\title{
Data in the time of COVID-19: A general methodology to select and secure a NoSQL DBMS for medical data
}

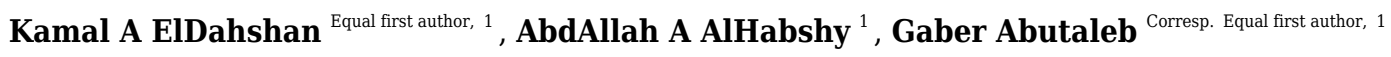 \\ ${ }^{1}$ Mathematics Department, Al Azhar University, Cairo, Egypt \\ Corresponding Author: Gaber Abutaleb \\ Email address: gaber_abutaleb@azhar.edu.eg
}

Background: As the COVID-19 crisis endures and the virus continues to spread globally, the need for collecting epidemiological data and patient information also grows exponentially. The race against the clock to find a cure and a vaccine to the disease means researchers require storage of increasingly large and diverse types of information; for doctors following patients, recording symptoms and reactions to treatments, the need for storage flexibility is only surpassed by the necessity of storage security. The volume, variety, and variability of COVID-19 patient data requires storage in a NoSQL database management systems (DBMSs). But with a multitude of existing NoSQL DBMSs, there is no straightforward way for institutions to select the most appropriate. And more importantly, they suffer from security flaws that would render them inappropriate for the storage of confidential patient data. Motivation: This paper develops an innovative solution to remedy the aforementioned shortcomings. COVID-19 patients, as well as medical professionals, could be subjected to privacy-related risks, from abuse of their data to communitarian bullying regarding their medical condition. Thus, in addition to being appropriately stored and analyzed, this their data must imperatively be highly protected against misuse. Methods: This paper begins by explaining the five most popular categories of NoSQL databases. It also introduces the most popular NoSQL DBMS types related to each one of them. Moreover, this paper presents a comparative study of the different types of NoSQL DBMS, according to their strengths and weaknesses. This paper then introduces an algorithm that would assist hospitals, and medical and scientific authorities to choose the most appropriate type for storing patients' information. This paper subsequently presents a set of functions, based on web services, offering a set of endpoints that include authentication, authorization, auditing, and encryption of information. These functions are powerful and effective, making them appropriate to store all the sensitive data related to patients. Results and contributions: This paper presents an algorithm to select the most convenient NoSQL DBMS for COVID-19 patients, medical staff, and organizations data. In addition, the paper proposes innovative security solutions Peerj Comput. Ści. reviewing PDF | (CS-2020:04:48359:2:0:NEW 24 Jul 2020) 
that eliminate the barriers to utilizing NoSQL DBMSs to store patients' data. The proposed solutions resolve several security problems including authentication, authorization, auditing, and encryption. After implementing these security solutions, the use of NoSQL DBMSs will become a much more appropriate, safer, and affordable solution to storing and analyzing patients' data, which would contribute greatly to the medical and research effort against COVID-19. This solution can be implemented for all types of NoSQL DBMSs; implementing it would result in highly securing patients' data and protecting them from any downfall related to data leakage 


\section{Data in the time of COVID-19: A general methodology 2 to select and secure a NoSQL DBMS for medical data}

3

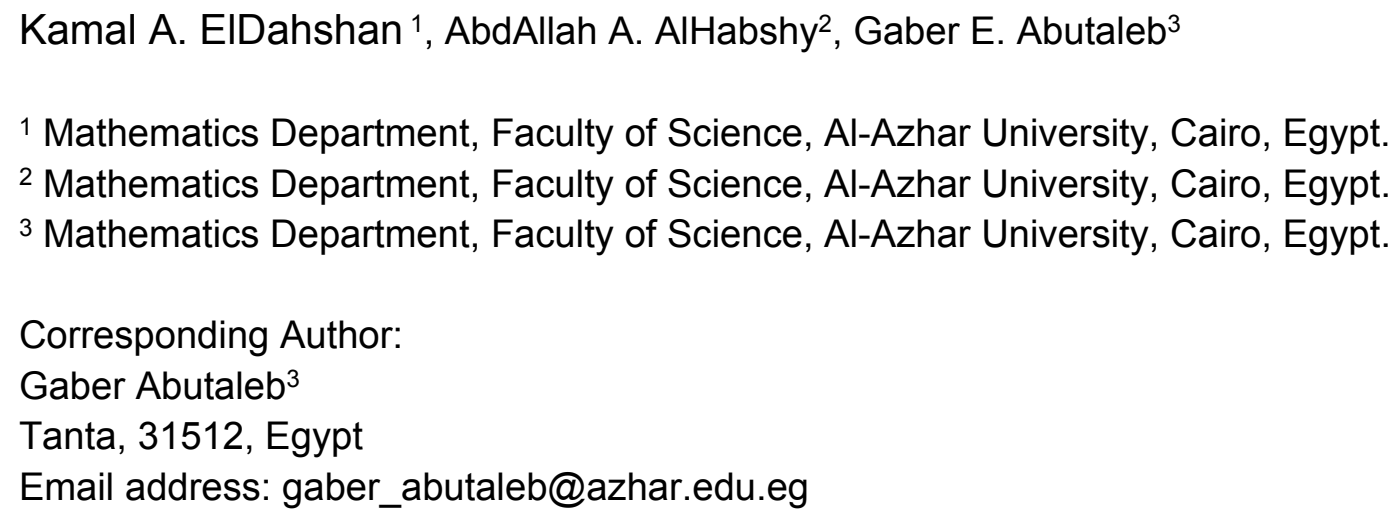

\section{Abstract}

Background: As the COVID-19 crisis endures and the virus continues to spread globally, the need for collecting epidemiological data and patient information also grows exponentially. The race against the clock to find a cure and a vaccine to the disease means researchers require storage of increasingly large and diverse types of information; for doctors following patients, recording symptoms and reactions to treatments, the need for storage flexibility is only surpassed by the necessity of storage security. The volume, variety, and variability of COVID-19 patient data requires storage in NoSQL database management systems (DBMSs). But with a multitude of existing NoSQL DBMSs, there is no straightforward way for institutions to select the most appropriate. And more importantly, they suffer from security flaws that would render them inappropriate for the storage of confidential patient data.

Motivation: This paper develops an innovative solution to remedy the aforementioned shortcomings. COVID-19 patients, as well as medical professionals, could be subjected to privacy-related risks, from abuse of their data to community bullying regarding their medical condition. Thus, in addition to being appropriately stored and analyzed, their data must imperatively be highly protected against misuse.

Methods: This paper begins by explaining the five most popular categories of NoSQL databases. It also introduces the most popular NoSQL DBMS types related to each one of them. Moreover, this paper presents a comparative study of the different types of NoSQL DBMS, according to their strengths and weaknesses. This paper then introduces an algorithm that would assist hospitals, and medical and scientific authorities to choose the most appropriate type for storing patients' information. This paper subsequently presents a set of functions, based on web services, offering a set of endpoints that include authentication, authorization, auditing, and encryption of information. These functions are powerful and effective, making them appropriate to store all the sensitive data related to patients. 
40 Results and contributions: This paper presents an algorithm to select the most convenient

41 NoSQL DBMS for COVID-19 patients, medical staff, and organizations data. In addition, the

42 paper proposes innovative security solutions that eliminate the barriers to utilizing NoSQL

43 DBMSs to store patients' data. The proposed solutions resolve several security problems

44 including authentication, authorization, auditing, and encryption. After implementing these

45 security solutions, the use of NoSQL DBMSs will become a much more appropriate, safer, and

46 affordable solution to storing and analyzing patients' data, which would contribute greatly to the

47 medical and research effort against COVID-19. This solution can be implemented for all types of

48 NoSQL DBMSs; implementing it would result in highly securing patients' data, and protecting

49 them from any downsides related to data leakage.

\section{1. Introduction}

51 The exponential increase in the number of patients infected with COVID-19, and the complex

52 race towards finding treatment and prevention protocols, is a concern shared by all. Across the

53 globe, researchers are collecting every shred of information related to the disease, and all data

54 related to patients and their relations, which brings along the challenge of recording large swaths

55 of information. The volume of data is expected to be immense - matched only by its diversity

56 and variability. And as the number of patients increases, and alongside it, the breadth and

57 diversity of data, medical organizations, researchers, and authorities will require storage systems

58 offering greater flexibility, and which aren't beholden to a strict set of options. As it stands, the

59 list of COVID-19 symptoms remains ill-defined; this entails the need for a database management

60 system (DBMS) that can handle unstructured data, namely, a NoSQL DBMS. But until now,

61 NoSQL database systems still struggle with security issues; the lack of security of NoSQL

62 DBMSs may make it an obstacle to its adoption as the preferred standard to store COVID-19

63 patients' personal data.

64 This paper begins by explaining the five most popular categories of NoSQL databases,

65 introducing the most popular NoSQL DBMS types under each. Features of NoSQL DBMS are

66 briefly explained, with a strong emphasis on security. The paper addresses security problems

67 such as authentication, authorization auditing, and encryption. Not only does the paper mentions

68 security vulnerabilities in each type, but it also lays out a comparative study of the different types

69 of NoSQL DBMS, compares the strengths and weaknesses of each. This comparison is built on a

70 set of criteria, namely Data Model, Owner Company, Open source(Y/N), Implementation

71 Programming Language, Stable Release issue dates, Support Authentication, Support

72 Authorization, Support Auditing, Support Data Encryption, and Query Types. The paper then

73 moves to provide an algorithm that would help organizations choose the most appropriate type

74 for storing COVID-19 patients' information.

75 The purpose of this paper is to propose security solutions that resolve this weakness of NoSQL

76 DBMSs, this eliminating the hurdle preventing medical organizations and other organizations

77 storing patient information from using them. The proposed solution solves several security

78 problems, including authentication, authorization, auditing, and encryption. The implementation

Peer] Comput. Sci. reviewing PDF | (CS-2020:04:48359:2:0:NEW 24 Jul 2020) 
79 of our security solutions will allow NoSQL DBMSs to become the more appropriate, safer, and

80 affordable solution to store and analyze COVID-19 patients' data.

\section{2. NoSQL SYSTEMS CATEGORIES AND THEIR SECURITY 82 ISSUES}

83 The NoSQL acronym was introduced in 2009, during an event on distributed databases

84 (Lakshman \& Malik, 2010). New technologies, such as Google's Bigtable (Chang \& Dean, 85 2008) were presented as being capable of managing large amounts of data. Research on NoSQL

86 databases has blossomed since (Lourenço \& Cabral, 2015). In addition to the five major 87 categories of NoSQL systems, some Multi-model NoSQL database systems, XML databases 88 (Elmasri \& Navathe, 2017), as well as other types of systems that have been available even 89 before the term NoSQL came into use, grew in popularity and usage. This section thus discusses 90 the five main categories of NoSQL systems, as well as the Multi-model database ones, with an 91 emphasis on their security features, in the interest of patient data safety. This section will also 92 address the most commonly-used NoSQL DBMSs in terms of security. NoSQL databases suffer 93 from a variety of security issues (Sahafizadeh \&Nematbakhsh, 2015). In NoSQL database 94 systems, security mechanisms such as Authentication and data encryption are either weak or 95 altogether inexistent.

96 First, we review possible attacks, and security mechanisms used to prevent them.

$97 \quad 2.1$ Introduction to Security Issues in NoSQL DBMSs

98

99

100

101

102

103

104

105

106

107

108

109

110

111

112

113

114

115

116

117

118

119

2.1.1 NoSQL Database Management Systems Classification. NoSQL database management systems are classified into five categories, namely Document-based Stores, keyvalue stores, Column-based or wide column Stores, Graph-based Stores, and Object Stores. Multi-model database categories may be added as shown in Fig. 1.

2.1.2 NoSQL DBMSs comparison criteria. Database management systems will be analyzed according to their basic information, security mechanisms, and potential attacks.

\section{- Basic information includes:}

- The owner and year of first appearance

- The classification, specifying whether it is open source or proprietary

- The DBMS' implementation programming language

- Some characteristics of the database management system

- The query language used in each database management system.

- Security mechanisms

- Authentication is the process of verifying the entity identity (user or device) permission to use the resources (data, application, etc.) (Zahid \& Masood, 2014).

- Verification is usually achieved through accessing control mechanisms, such as users' passwords and certificates (Grolinger \& Higashino, 2013).

- Authorization is the mechanism that ensures that only an authorized user is allowed to access system resources. Authorization is usually performed with the user's permission (Grolinger \& Higashino, 2013).

- Auditing is the process of keeping track and recording all actions performed by database users (Elmasri \& Navathe, 2017). 
- Data Encryption refers to protecting and encrypting sensitive data throughout the communications network so it cannot be read by unauthorized users or attackers (Elmasri, 2017; Grolinger, 2013). There are three levels of encryption solution; Data at rest, Client-to-server communication, and Server-to-server connections (Grolinger \& Higashino, 2013).

- Attacks: some attack types that DBMSs may endure include:

- Script Injection, wherein a security vulnerability is used by an adversary to inject malicious code in pages or web forms to damage or retrieve data (Sullivan, 2011; Jim, 2007).

- SQL Injection, which may occur when an adversary injects a string input through the application to damage, change the database, retrieve the sensitive data, and run commands to deny service (Elmasri \& Navathe, 2017).

- Denial of service (DOS), in which an adversary floods the server with requests, thus preventing legitimate users from accessing the service, or the attacker may delete some data (Elmasri \& Navathe, 2017).

- An inactive connection may occur when an adversary opens the connection for a long time.

\subsection{Document-Based NoSQL Stores}

Document-oriented, or document-based NoSQL systems store data as collections of similar documents. These types of systems are also known as document stores (Moniruzzaman \& Hossain, 2013; Dindoliwala, Morena, 2017). Each document is similar to a row in relational databases, but is more flexible because it has no schema (is schema-free) and is specified as selfdescribing data (Elmasri \& Navathe, 2017). The users or the processing applications have the schematic responsibility in document databases. This may result in some disadvantages, such as the lack of referential integrity and normalization. However, the lack of schema provides high flexibility to store a wide range of data, which makes it suitable for stores of big data. In document-based databases, a record, called a document, has its own internal structure, and can be stored as a value for every key (document ID). Documents are separated from one another, but are grouped as a collection of data, and the database ensures that each query receives the document version with the largest number of changes. Since this cannot guarantee full concurrency control, it is called eventual consistency. The consistency takes some time to achieve, which significantly speeds up the data processing at the expense of transactional security. In document-based databases, queries can be performed in parallel and, therefore, sped up with the map-reduce procedure (Meier \& Kaufmann, 2019). Document-based databases are also fit for storing sparse data, or semi-structured data, but that would necessitate an extensive use of Nulls for data completeness purposes (Moniruzzaman \& Hossain, 2013). Documents can be specified in various formats, such as XML (Extensible Markup Language), YAML (Yet Another Markup Language) (Kaur \& Rani, 2013), or BSON (Binary JSON (JavaScript Object Notation) (Moniruzzaman \& Hossain, 2013; Özsu \& Valduriez, 2020). The popular language to specify documents in NoSQL systems is JSON (Han \& Haihong, 2011). Document-Based NoSQL Systems are suitable for managing Big Data collections of literal documents, like XML documents, email messages, text documents, online shopping, event logging, deep analytical processing, and content management. Examples of Document-Based NoSQL DBMSs (database management systems) include CouchDB (JSON), MongoDB (BSON) (Özsu \& Valduriez, 2020), Terrastore, ThruDB, OrientDB, RavenDB, Citrusleaf, SisoDB, CloudKit, Perservere and Jackrabbit) (Tudorica \& Bucur, 2011). 
166 Security Issues in Document-Based NoSQL DBMSs

167 This subsection discusses the most popular types of document Stores NoSQL Database

168 Management Systems.

169 2.2.1 MongoDB

170

171

- Introduction:

172

173

174

175

176

177

178

179

180

181

182

183

184

185

186

187

188

189

190

191

192

193

194

195

196

197

198

199

200

201

202

203

204

205

206

207

208

209

210

211

MongoDB is an open-source document-oriented NoSQL DBMS. It is a schema-free, highly available, fault-tolerant and scalable NoSQL DBMS. MongoDB supports sharding by configuring shared clusters (Zahid \& Masood, 2014). It was developed by MongoDB Inc., first appearing in 2009; its stable release appeared in June 2018. MongoDB was written in C++, C, and JavaScript (Nayak \& Poriya, 2013, Özsu \& Valduriez, 2020), and it consists of one or more collections of documents. A collection is analogous to a table, but has no pre-defined schema. The document, which is the data storing unit in MongoDB, has an ID and is equivalent to a record in the relational DB. Insert, delete, and update operations can be performed on a collection (Dindoliwala \& Morena, 2017).

Mongo Query Language is the query language used By MongoDB DBMS to manipulate certain documents from a database collection. Map-reduce and REST querying are supported by MongoDB (Chodorow, 2013).

- Security Analysis:

- Authentication: granting to users through the database itself or integration with an external mechanism like LDAP (Lightweight Directory Access Protocol). MongoDB grant Authentication to entities by using the SCRAM-SHA-1 IETF standard (Zugaj \& Beichler, 2019). An authentication command is used to authenticate the users, whereas database-nodes are authenticated to the MongoDB cluster via key files. The default Authentication mechanism in MongoDB is SCRAM-SHA-1, which verifies the supplied user credentials with the name of the user, user's password, and Authentication database. With LDAP, MongoDB can authenticate and authorize users directly via corporate LDAP infrastructure to enforce centralized access policies (Dindoliwala \& Morena, 2017). MongoDB doesn't support authentication, however, if running in shared mode (Sahafizadeh, 2015 \& Noiumkar, 2014). When the authentication in MongoDB is based on the key or the password, it's called a pre-shared secret. This password has been hashed with the MD5 algorithm before being stored in the key file. Hackers can crack the preshared secret's hashed value by cracking the MD5 (Dindoliwala \& Morena, 2017), because the MD5 algorithm is not secured (Sahafizadeh \& Nematbakhsh, 2015). MongoDB uses SSL with X.509 certificates to secure communication between the user and the MongoDB cluster and intra-cluster authentication (Zahid \& Masood, 2014).

- Authorization: Administrators in MongoDB can determine the permissions for users or applications. They can also decide what data can be seen by users or applications when performing a database query. The authorization mechanism in MongoDB contains LDAP authorization, roles, and Field-Level Security with Read-Only Views. In MongoDB privileges are assigned to roles, then roles are assigned to users. MongoDB involves built-in roles that are used by administrators to control access to the MongoDB system. A privilege consists of a specified resource and actions permitted on this resource. A resource is a collection, set of collections, databases, or a cluster. MongoDB supports a simple role-based authentication system that allows administrators to decide who has access to the database and which level of access they have. Furthermore, MongoDB supports authorization through LDAP (Dindoliwala \& Morena, 2017). 
212 - Auditing: Auditing framework stores actions such as operations that have been performed

213 by the users in the database, and authentication and authorization activities along with write and read operations on the database. Administrators can extract and filter audit trails for any operation with MongoDB. For example, it is possible to audit and log the identities of users who accessed specific documents and the changes they made to the database during their session. Administrators can configure MongoDB to log all the actions or specific actions. MongoDB Enterprise Advanced (Dindoliwala \& Morena, 2017) also supports the role-based auditing. With MongoDB, it is possible to report the DB activities as well.

- Data Encryption: in MongoDB, data can be encrypted over the network, in backups, and the rest in permanent storage. MongoDB Enterprise Advanced supports FIPS 140-2 encryption when it run in FIPS Mode (Dindoliwala \& Morena, 2017). In MongoDB, the data files are not encrypted automatically, so all data is stored as plaintext. This means that any malicious user with access to the file system can extract information from the files. MongoDB uses RESTful to manage its server via the HTTP protocol. However, there is no data encryption for these ports, which means that hackers can display, capture and get access to the data since no data encryption was performed while the data was being sent back and forth between the client and server (Noiumkar, 2014).

- Script Injection: Using JavaScript, hackers can attack MongoDB. Examples about the attacks that occurred on MongoDB can be found in this reference (Jim, 2007). NoSQL databases are exposed to injection attacks like SQL databases. Son presented four examples of GitHub site for injection vulnerabilities in PHP MongoDB-based applications (Son, 2013).

\subsubsection{CouchDB}

\section{- Introduction:}

CouchDB is an open-source NoSQL DBMS. It was developed using Erlang (George, 2013). It became an Apache project in 2008. It was released in 2005 and the stable release appeared in November 2017. CouchDB runs on Hadoop Distributed File Systems (HDFS). CouchDB is document-based NoSQL DBMS, fault-tolerant, highly scalable, available, and flexible (Sahafizadeh \& Nematbakhsh, 2015). It supports document redistribution across nodes by cluster configuration for large performance improvements. A cluster uses the phenomenon of incremental replication by periodically copying any changes in a document on a single node to other nodes. The incremental replication results in inconsistency and data redundancy in the cluster. CouchDB uses the JavaScript, JSON, and SQL++ as its query language (Özsu \& Valduriez, 2020). Map-reduce and REST querying are supported by CouchDB as well (Noiumkar, 2014).

- Security Analysis:

- Authentication: CouchDB uses a process called CRUD to perform authentication (Noiumkar, 2014). The authentication in CouchDB is based on both cookies and passwords. It uses the PBKDF2 hash algorithm to encrypt the password and send it over the network using SSL protocol (Sahafizadeh, 2015 \& Zahid, 2014).

- Authorization: There are three levels of users within the CouchDB; database admin, server admin, and database member (Grolinger \& Higashino, 2013). Authorization is implemented only at the database level where only a single role in access control is supported (Zahid \& Masood 2014). 
257

258

259

260

261

262

263

264

265

266

267

268

269

270

271

272

273

274

275

276

277

278

279

280

281

282

283

284

285

286

287

288

289

290

291

292

293

294

295

296

297

298

299

300

301

- Auditing: The level of auditing in CouchDB is moderate (logging of all changes to userprofiles and the level of database). Auditing is provided to log events and views in log files. However, CouchDB does not support automatic backups of database logs, replicas, and automatic logging; the configuration of logs is thus the responsibility of the database administrators (Zahid \& Masood 2014).

- Data Encryption: CouchDB does not have automatic data encryption, so the data files are at risk of being accessed and read directly (Sahafizadeh, 2015 \& Noiumkar, 2014). Data Encryption is supported in Client/Server via SSL. Also, data encryption could be supported using HTTPS connections in Server/Server (Grolinger \& Higashino 2013).

- Denial of service attack: Websites such as securityfocus.com display vulnerabilities that exist only on Apache CouchDB 1.5.0 (SecurityFocus, 2020). These vulnerabilities are used by hackers to crash it. So CouchDB is potentially vulnerable to Denial of service attacks (Sahafizadeh \& Nematbakhsh, 2015).

- Script Injection: CouchDB uses JSON to manipulate the data, making it vulnerable to Script Injection. In other words, hackers can launch JSON Injection to attack CouchDB (Sahafizadeh, 2015 \& Noiumkar, 2014).

\subsubsection{Couchbase Server}

- Introduction:

Couchbase Server is an open-source, document-oriented NoSQL DBMS. Couchbase Server was developed by Couchbase Inc., initially released in August 2010 with the stable release launched in February 2018. Couchbase Server was developed using Erlang, $\mathrm{C}$, and $\mathrm{C}++$ (George, 2013). Various data centers contain all the cluster servers. The documents are stored in vbuckets, which are special data containers uniformly distributed across the cluster. More nodes can be added and removed because Couchbase cluster scales are completely horizontal (Zahid \& Masood, 2014). Map-reduce and REST querying is supported by the Couchbase server (Grolinger \& Higashino, 2013). Couchbase also supports SQL++, an SQL like language which has been designed to become a unified query language for NoSQL system.

- Security Analysis:

- Authentication: SASL (Simple Authentication and Security Layer) is supported by name and password on Couchbase Server HTTP. Basic authentication is used in Couchbase management REST (Representational State Transfer) API (Application Programming Interface) (Grolinger \& Higashino, 2013). External Authentication is also supported (Zahid \& Masood 2014).

- Authorization: Couchbase Server does not support authorization (Grolinger \& Higashino, 2013).

- Auditing: Couchbase Server does not support auditing (Grolinger \& Higashino, 2013).

- Data Encryption: Couchbase Server does not support data Encryption in Data at rest, Client/Server, and Server/Server (Grolinger \& Higashino, 2013).

\subsection{Key-Value NoSQL Stores}

These are the simplest NoSQL databases. Key-value stores focus on high availability, performance, and scalability, by storing data in a distributed storage system (Abed, 2020). The data model used in key-value stores is simple, and in most such systems, there is no query language, but rather a set of operations that can be used by the application programmers. The key is a unique identifier associated with a value, and is used to rapidly locate it (Kaur \& Rani, 
302

303

304

305

306

307

308

309

310

311

312

313

314

315

316

317

318

319

320

321

322

323

324

325

326

327

328

329

330

331

332

333

334

335

336

337

338

339

340

341

342

343

344

345

2013). The value may have different formats for different key-value storage systems. In some cases, the value is just an array or a string of bytes. Key-value stores are suitable for efficient reading and writing of extensive amounts of data. Some disadvantages of this model include the lack of some traditional capabilities such as atomicity and consistency, and as the data volume increases, retaining more values as keys may become more difficult (Dindoliwala \& Morena, 2017). The application using the key-value store has to interpret the structure of the data value. Different key-value stores can thus store structured, unstructured, or semi-structured data items (Elmasri \& Navathe, 2017). NoSQL Key-Value stores are needed for application tasks such as managing user profiles, sessions, or retrieving product names (Moniruzzaman \& Hossain, 2013). Examples of this type of NoSQL DBMSs include Redis, Voldemort (LinkedIn), Riak, and BerkeleyDB (Özsu \& Valduriez, 2020).

\section{Security Issues in Key-Value Stores NoSQL DBMSs}

This subsection discusses the most popular types of Key-Value Stores NoSQL Database Management Systems.

\subsubsection{Redis}

- Introduction:

Redis is an Open source NoSQL DBMS (Grolinger \& Higashino, 2013), developed using $\mathrm{C}$ and C++ (Meier \& Kaufmann., 2019) by Salvatore Sanfilippo. Redis was initially released in May 2009 and the stable release appeared in June 2018. It is a Key-value memory database, So, Redis data will be loaded into memory (Dayan, 2018) when it runs and all operations are run in memory. Redis periodically saves data asynchronously to the hard disk. It achieves high performance thanks to its use of pure memory. Redis can handle more than 100,000 write or read operations per second. The maximum limit of value is $1 \mathrm{~GB}$. However, Redis cannot be used as big data storage, and scalability is poor because the capacity of the Database is limited by the physical memory. As such, Redis is suitable for providing high-performance computing for a small amount of data (Han \& Haihong, 2011). SQL-like and Map-reduce querying is not supported in Redis but REST querying is supported by third-party APIs (Grolinger \& Higashino, 2013).

- Security Analysis:

- Authentication: Redis provides a password-based authentication to its clients. Passwords are set by system administrators and stored in plaintext format. Redis does not provide default authentication and listens on all IP addresses on port 6739(Han \& Haihong, 2011).

- Authorization: Redis does not support any kind of access control (Han \& Haihong, 2011) i.e. it does not support authorization (Paterson, 2006).

- Auditing: Redis does not provide support for auditing (Tudorica, 2011; Paterson, 2006).

- Data Encryption: Redis does not provide support for Data encryption. It stores data in plaintext (Paterson, 2006). As such, hackers - or anyone who can access the Redis server - will be able to read all the data in the database. No data encryption is performed in the communication between client and server, and between other servers, neither at the same cluster nor different clusters (Nayak \& Poriya, 2013). Redis does not have data-at-rest encryption (Zugaj \& Beichler, 2019). 
346

347

348

349

350

351

352

353

354

355

356

357

358

359

360

361

362

363

364

365

366

367

368

369

370

371

372

373

374

375

376

377

378

379

380

381

382

383

384

385

386

387

388

389

- Denial of service attack: Denial of service attacks on Redis was not reported (Nayak \& Poriya, 2013).

- Script Injection: Redis does not have a concept of string escaping, so injection becomes impossible (Nayak, 2013, Paterson, 2006).

\subsubsection{Voldemort}

- Introduction:

Voldemort is an Open source key-value NoSQL DBMS, developed by LinkedIn (Dayan, 2018). Its initial release was in 2009 and the stable release appeared in July 2017 (Grolinger \& Higashino, 2013). Voldemort was developed using Java (Deka, 2013). Three simple operations are included in Voldemort; reading, writing, and deletion. All of them are executed using a key (Chen, 2014). There is no SQL-like querying language in Voldemort but Map-reduce querying is supported (Grolinger \& Higashino, 2013).

- Security Analysis:

- Authentication: There is no authentication mechanism in Voldemort.

- Authorization: There is no authorization mechanism in Voldemort.

- Auditing: There is no auditing in Voldemort.

- Data Encryption: No encryption, neither between the client and the server nor among the servers (Grolinger \& Higashino, 2013).

\subsubsection{Oracle Berkeley DB (BDB)}

- Introduction: Oracle Berkeley DB is a closed source key-value NoSQL DBMS. Oracle Berkeley DB was developed by Sleepycat Software. Oracle Berkeley DB initial release appeared in 1994 and the stable release was in June 2018. It is a high-performance data management services to applications. It uses a simple function for data access and management (Sullivan, 2011). SQLite and REST querying is supported by Oracle Berkeley DB, but Map-reduce querying is not (Grolinger \& Higashino, 2013). In terms of implementation of Oracle Berkeley DB, was implemented in C, Oracle Berkeley XML was implemented in $\mathrm{C}++$ and Oracle Berkeley JE was implemented in Java.

- Security Analysis:

- Authentication: There is no authentication mechanism in Berkeley DB.

- Authorization: There is no authorization mechanism in Berkeley DB.

- Auditing: There is no auditing in Berkeley DB.

- Data Encryption: Berkeley DB supports data at rest encryption. No encryption between Servers (Grolinger, Higashino 2013).

\subsection{NoSQL Graph Stores}

A graph database is a database that uses graph structures for semantic queries with nodes, edges, and properties to represent and store data. A key concept of this system is the graph, which relates data items in the store. Both edges and nodes can be labeled to indicate the types of vertices and associations they represent, and it is generally possible to store data associated with both individual nodes and individual edges (Elmasri \& Navathe, 2017). In the Graph database model, the database is represented as a network structure containing edges between nodes to illustrate the relationships among nodes. Nodes may also contain properties that describe the real data contained within each object. Edges may also have properties. An edge (or a relationship) connects two nodes and it may be directed. When directions are added, relationships between

Peer) Comput. Sci. reviewing PDF | (CS-2020:04:48359:2:0:NEW 24 Jul 2020) 
390

391

392

393

394

395

396

397

398

399

400

401

402

403

404

405

406

407

408

409

410

411

412

413

414

415

416

417

418

419

420

421

422

423

424

425

426

427

428

429

430

431

432

433

nodes are identified by the names of the nodes, and can be traversed in both directions (Kaur \& Rani, 2013). The graph uses the Index-Free adjacency technique, in which each node contains a pointer pointing to the adjacent node. For every node in the Graph database, the system can access its direct neighbor, without the need to consider all edges (Abed, 2020). This is known as the index-free adjacency property; and it is the key feature of the graph databases (Meier \& Kaufmann, 2019). Graph databases are efficient schema-less DBs, which makes them suitable for semi-structured data storage. The queries in Graph databases are expressed as traversals; which make them much faster than relational databases (Nayak \& Poriya, 2013). In general, graph databases are appropriate when the main interest is the relationships between data (e.g., generating recommendations, social networks, conducting forensic investigations, network search, and fraud detection (Moniruzzaman \& Hossain, 2013). Examples of Graph Databases include Neo4j, Sones GraphDB, InfiniteGraph, InfoGrid, and AllegroGraph (Khasawneh, ALSahlee, 2020).

\section{Security Issues in Graph NoSQL DBMSs}

This subsection discusses the most popular types of graph Stores NoSQL Database Management Systems.

\subsubsection{Neo4j}

- Introduction:

Neo4j is an open-source graph NoSQL DBMS (Paterson, 2006), developed by Neo Technology. It was initially released in 2007 and the stable release appeared in April 2018. It was developed using Java. The advantages of Neo4j include providing objectoriented, flexible network structure, reliability, highly available, and scalability. Neo4j uses the graph data model which consists of nodes and edges and the relationships between them. Neo4 $j$ uses CYPHER query language. REST querying is supported by Neo4j but Map-reduce is not supported (Grolinger \& Higashino, 2013). Neo4j is used extensively in software that has complex relationships such as social networking and recommendation engines (Moniruzzaman \& Hossain, 2013).

- Security Analysis:

- Authentication: Neo4j does not support Authentication (Grolinger \& Higashino, 2013).

- Authorization: Neo4j does not support authorization (Grolinger, 2013; Paterson, 2006)

- Auditing: Neo4j does not support auditing (Grolinger, 2013; Paterson, 2006).

- Data Encryption: Neo4j does not support data at rest encryption, nor does it support data encryption between servers (Grolinger, Higashino, 2013); however it uses SSLbased communication protocol between the client and the server (Paterson, 2006).

\subsubsection{HyperGraphDB}

- Introduction:

HyperGraphDB is an Open source NoSQL DBMS that implements powerful knowledge management formalism known as directed hypergraphs (Grolinger, Higashino, 2013). HyperGraphDB is developed by Kobrix Software, Inc. The stable release of HyperGraphDB appeared in May 2017. Hyper-GraphDB was developed using Java. Higher-order Relations are naturally represented by the HyperGraphDB model. The HyperGraphDB model is very useful in areas related to data modeling such as artificial 
434

435

436

437

438

439

440

441

442

443

444

445

446

447

448

449

450

451

452

453

454

455

456

457

458

459

460

461

462

463

464

465

466

467

468

469

470

471

472

473

474

475

476

477

intelligence and knowledge of representation and bioinformatics. This data model is designed for AI, Knowledge representation and the semantic Web (Kobrix, 2020; Angles, 2012). HyperGraphDB provides SQL- like query language. REST querying is supported by HyperGraphDB but Map-reduce is not (Grolinger \& Higashino, 2013).

- Security Analysis:

- Authentication: HyperGraphDB does not support authentication.

- Authorization: HyperGraphDB does not support authorization.

- Auditing: HyperGraphDB does not support auditing.

- Data Encryption: HyperGraphDB does not support data encryption (Grolinger \& Higashino, 2013).

\subsubsection{AllegroGraph}

- Introduction:

AllegroGraph is a closed-source graph NoSQL DBMS, developed by Franz Inc. The stable release appeared in October 2017 (Khasawneh \& AL-Sahlee 2020; Grolinger \& Higashino 2013). It was developed using Common Lisp (Franz, 2020). It offers highperformance and is mainly used for developing semantic web applications. It can store data and meta-data as RDF (Resource Description Framework) triples. AllegroGraph maintains high performance when accessing billions of quads due to efficient memory utilization along with disk-based storage (Arora, 2013; Franz, 2020). SPARQL and Prolog are the query languages used by AllegroGraph. REST querying is supported by AllegroGraph but Map-reduce is not (Grolinger \& Higashino, 2013).

- Security Analysis:

- Authentication: Authentication is supported by AllegroGraph.

- Authorization: Authorization is supported by AllegroGraph such as read, write, and delete.

- Auditing: AllegroGraph can be used to record specific changes in the audit log.

- Data Encryption: AllegroGraph does not support data at rest encryption. But it uses an HTTPS-based communication protocol between the client and the server (Grolinger \& Higashino 2013).

\subsection{Column-Based or Wide Column NoSQL Stores}

By providing an additional structure, Column-based databases utilize the key-value concept. In certain uses, it has been proven that to optimize the read operations, it is much better to store the data into the relational datasets per column, not per row. This is due to the occasional need for all columns in a single row at once; however, there are groups of columns that are often read together (Meier \& Kaufmann, 2019). Column-based data stores are designed to address the huge number of columns and frequent changes in the schema (Moniruzzaman \& Hossain, 2013). Wide column stores have tables that contain columns. A column is specified by a combination of a Column Family and a Column Qualifier; each column family has a name that should be declared when the table is created, and cannot be changed subsequently. After loading data into a table, each column family is associated with column qualifiers. Column qualifiers are not declared when the table is created, but can be dynamically created and inserted into the table. Read and write are done using columns rather than rows. The reason for structuring the data in groups of columns - column families - as storage units is that it is a sound way to optimize the data access. Column-based databases adopt this model; they store the data not in relational datasets but rather

Peer] Comput. Sci. reviewing PDF | (CS-2020:04:48359:2:0:NEW 24 Jul 2020) 
478 in enhanced and structured multi-dimensional keyspaces (Meier \& Kaufmann, 2019). Wide479 column stores have great features such as high performance and high scalability (Elmasri \& 480 Navathe, 2017). These types of DBMSs are suitable for data processing, distributed data storage, 481 content management, event logging, and categorizing for analytics (Moniruzzaman \& Hossain, 482 2013). The most common open-source column-oriented databases are HBase, Hypertable. HBase 483 are derivatives of Bigtable (Kaur \& Rani, 2013).

484 Security Issues in Column-based NoSQL DBMSs

485 This subsection discusses the most popular types of column-based Stores NoSQL Database 486 Management Systems.

\subsubsection{Hypertable}

- Introduction: Hypertable is a column-oriented NoSQL DBMS. It was developed by Zvents before 2008 and the stable release appeared in March 2016. Hypertable was developed using $\mathrm{C}++$ (Deka, 2013). It is a high-performance open-source DBMS, and can run on Hadoop Distributed File Systems (HDFS). It is modeled after Google's Bigtable DBMS. Like Bigtable, Hypertable stores data using the column-oriented methodology (Khasawneh, AL-Sahlee, 2020). There is no SQL-like query language in Hypertable.

- Security Analysis:

- Authentication: Hypertable does not support Authentication (Nayak, 2013; Paterson, 2006). Moreover, it does not support Authentication in communication between the client and the server or among its servers (Nayak, Poriya 2013).

- Data Encryption: Hypertable does not support encryption for its data files (Nayak, 2013, Paterson, 2006). Hypertable does not support encryption in communication between the client and server or between its servers (Nayak \&Poriya 2013).

- Denial of service attack: There is no information reported about denial of service attacks on Hypertable.

- Script Injection: Though Hypertable has an HQL (Hypertable Query Language), which is similar to the SQL, it is not vulnerable to script injection (Nayak \& Poriya 2013).

\subsubsection{HBase}

- Introduction:

HBase is an open-source column-oriented NoSQL DBMS. HBase was developed by the Apache Software Foundation. Its initial release was in February 2015 and the stable release was in April 2018. It was developed using Java (Meier \& Kaufmann, 2019). Features include automatic distributability and scalability. HBase is based on the concept of Google's Bigtable and implemented in Java (Tudorica, 2011; Paterson, 2006). There is no SQL-like query language in HBase, but Map reduce and REST querying are supported (Grolinger, Higashino, 2013).

- Security Analysis:

- Authentication: HBase supports token-based Authentication, for MapReduce tasks and user Authentication is done by using SASL (Simple Authentication and Security Layer with Kerberos (Tudorica, 2011; Paterson, 2006).

- Authorization: HBase supports authorization by access control list. Permissions include create, read, write and admin (Grolinger, 2013; Paterson, 2006).

- Auditing: Auditing is supported by HBase (Han \& Haihong, 2011). 
522

523

524

525

526

527

528

529

530

531

532

533

534

535

536

537

538

539

540

541

542

543

544

545

546

547

548

549

550

551

552

553

554

555

556

557

558

559

560

561

562

563

564

- Data Encryption: HBase does not support the encryption of data at rest (Tudorica, 2011; Paterson, 2006), but HBase supports the encryption in communication between client and server (Grolinger \& Higashino 2013).

- Denial of service attack: No report for denial of service attack.

- Script Injection: No report for script injection (Paterson, 2006).

\subsection{Object Store NoSQL Stores}

An object-oriented database stores the information or data as objects according to the objectoriented paradigm (Nayak \& Poriya, 2013). Object-oriented stores can be thought of as a combination of object-oriented programming (OOP) methodology and database principles. Data encapsulation, polymorphism, inheritance, and all other features of OOP are offered by other object-based databases. The classes, attributes, and objects in the Object-oriented stores are comparable to tables, columns, and tuples in a table in RDBMS respectively. Each object has an identifier that uniquely represents it. Because the object can easily be retrieved using pointers, data access becomes much faster in object-oriented databases. Harnessing the Object-based databases can ease the agility of modern software development processes (Nayak \& Priya, 2013). These types of data stores are not relational databases and are not queried using SQL (Dindoliwala \& Morena, 2017). Object Stores are the most convenient category for storing and retrieving binary large objects such as files, images, videos, and audio files. They are ideal for applications that require complex relationships between objects and changing object structures, or if the application defines members that are grouped in collections. Common uses for the object-oriented databases are applications of scientific research, telecommunication, computeraided drafting, etc. The disadvantage of object-oriented databases is the limited programming languages they are bounded to (Nayak \& Poriya 2013). Examples of Object Store NoSQL DBMS' include Gemstone, db4o, and objective/DB (Dindoliwala \&Morena 2017).

\section{Security Issues in Object Store NoSQL DBMSs}

This subsection discusses the most popular types of object Stores NoSQL Database Management Systems.

\subsubsection{Gemstone}

- Introduction: Gemstone is an open-source an Object NoSQL DBMS. It was developed by GemTalk Systems. Its first appearance was in 1986 and the stable release is 64 Bit 3.3.7. Gemstone is Proprietary commercial software. Multiuser environments are supported by Gemstone; each user can have one or more sessions, and multiple user sessions can be active at the same time. Gemstone provides security at several levels from login authorization to object access (Dindoliwala \& Morena, 2017). There is no general query language like SQL in Gemstone.

- Security Analysis:

- Authentication: In Gemstone, each user is identified by a unique user ID and a password. The user is represented by an instance of the class User Profile. The User Profile contains a user ID, password, default authorization information, and the user's group. Only users who have a User Profile can logon to the system.

- Authorization: Authorization exists within Gemstone and controls individual object access. 
565

566

567

568

569

570

571

572

573

574

575

576

577

578

579

580

581

582

583

584

585

586

587

588

589

590

591

592

593

594

595

596

597

598

599

600

601

602

603

604

605

606

607

608

- Auditing: The DB administrator can also configure the Gemstone system to monitor failures to login.

- Data Encryption: Gemstone does not support Data Encryption (Dindoliwala \& Morena, 2017).

\subsection{2 db4o}

- Introduction: $\mathrm{db} 4 \mathrm{o}$ is an open-source object NoSQL DBMS. It was developed by Action and the stable release 8.0. db4o was developed using Java and C\# (Noiumkar, 2014). db4o-SQL is an interface to allow SQL queries into a db4o database.

- Security Analysis:

- Authentication: db4o provides only an internal authentication mechanism.

- Authorization: db4o offers file-level authorization.

- Auditing: db4o lacks auditing capabilities.

- Data Encryption: db4o uses the eXtended Tiny Encryption Algorithm (Dindoliwala \& Morena, 2017) for data encryption.

\subsubsection{Objectivity/DB}

- Introduction: Objectivity/DB is an object NoSQL DBMS. Objectivity/DB is proprietary to Objectivity Inc. and first commercialized in 1990. Objectivity/DB was developed using Java, C\#, $\mathrm{C}++$, and Python. Since the Objectivity/DB schema is created from internal class definitions from the programming language, it is easier to maintain changes to the schema (Dindoliwala \& Morena, 2017). Objectivity/DB provides OQL (Object Query Language which is like SQL querying language

- Security Analysis:

- Authentication: For Authentication Objectivity/DB has two mechanisms; the first (by default) is Kerberos; the second is by using Advanced Multithreaded Server (AMSprotocol).

- Authorization: Relies on the operating system and file systems for access control.

- Auditing: No auditing capabilities have been mentioned in Objectivity/DB.

- Data Encryption: Objectivity/DB doesn't provide any inbuilt encryption (Dindoliwala \& Morena, 2017).

\subsection{Multi-model NoSQL database Stores}

This term refers to at least two types of NoSQL categories integrated into one system. In some of the NoSQL systems, a combination of more than one NoSQL system is used. This integration ensures that all the features in each category are combined. For example, Cassandra is a NoSQL DBMS used on Facebook. It combines column stores and key-value stores (Nayak \& Poriya, 2013). DynamoDB is a NoSQL database used in Amazon. It combines document and key-value data models (Sahafizadeh \& Nematbakhsh, 2015).

\section{Security Issues in Multi-model NoSQL DBMSs}

This subsection discusses the most popular types of Multi-model database Stores NoSQL Database Management Systems.

\subsubsection{Cassandra}

- Introduction: 
609

610

611

612

613

614

615

616

617

618

619

620

621

622

623

624

625

626

627

628

629

630

631

632

633

634

635

636

637

638

639

640

641

642

643

644

645

646

647

648

649

650

651

652

653

654

Cassandra is an open-source Multi-model database NoSQL DBMS. It was developed by Apache Software Foundation and its stable release appeared in February 2018. Cassandra was developed using Java, and is used by Facebook. Cassandra is a Multi-model database of both column stores and key-value stores (Moniruzzaman \& Hossain, 2013). Its key characteristics include: a free schema, flexibility, convenience of adding or deleting fields, support for range queries, partition tolerance, and high scalability (Han \& Haihong, 2011). Cassandra has three basic components; data, centers, and clusters nodes. The data in a cluster is organized into key spaces (databases), which contain tables. Tables contain rows, and rows have columns. The query language used is Cassandra Query Language (CQL) also Cassandra supports Map-reduce querying and REST querying by Third-party APIs (Dindoliwala \& Morena, 2017).

- Security Analysis:

- Authentication: authentication in Cassandra is particularly weak since passwords are encrypted using the MD5 hash. The entire authentication in Cassandra is provided between the client and the Cassandra cluster. In other words, the inter-node message exchange does not support Authentication by default. Hence, a malicious user with access to the network used by the Cassandra cluster can bypass the client-side Authentication and extract data or damage it. However, the transmission security at the data center, rack, and a cluster is provided by Cassandra via enabling SSL/TLS in its configurations (Han \& Haihong, 2011). Cassandra supports only internal Authentication mechanisms. Cassandra 3.0 Authentication is role-based and stored internally in Cassandra system tables (Dindoliwala \& Morena, 2017).

- Authorization: The entire authorization mechanism in Cassandra is provided between the client and the Cassandra cluster (Han \& Haihong, 2011). As such, a malicious user with access to the network used by the Cassandra cluster can bypass the client-side authorization and extract data or damage it. In 2015, it had been proven that Cassandra did not support authorization (Sahafizadeh \& Nematbakhsh 2015; Paterson, 2006). Cassandra uses the GRANT/REVOKE mechanism to manage permissions as part of the authorization mechanism. In Cassandra 2.2 and later versions, Role-based access control is available and permissions may be applied on resources such as keyspace, table, function (Dindoliwala \& Morena, 2017).

- Auditing: Cassandra does not support auditing (Tudorica, 2011; Paterson, 2006). Cassandra auditing is available in Enterprise Cassandra as a log4jbased integration and a per-node basis. Filters are available for logging using a combination of the following categories - ADMIN, AUTH, ALL, DCL, DML, and QUERY (Dindoliwala \& Morena, 2017).

- Data Encryption: Data files in Cassandra are stored without encryption and the database does not have automatic data encryption, so malicious users can access the data and read it (Nayak, 2013; Tudorica, 2011). Since version 3.2, Cassandra supports at-rest data encryption through Transparent Data Encryption (TDE) (Dindoliwala \& Morena, 2017). - SQL Injection: Cassandra Query Language (CQL) has a similar syntax to SQL's, so it is believed that it can be attacked in the same way SQL is vulnerable to attacks by SQL injection (Nayak, 2013; Paterson, 2006).

- Denial of service attack: Cassandra performs one thread per client. As such, it is vulnerable to denial of service attacks. A malicious user creating enough fake connections can drain Cassandra's resources. If hackers knew the IP addresses of all

Peer] Comput. Sci. reviewing PDF | (CS-2020:04:48359:2:0:NEW 24 Jul 2020) 
655

656

657

658

659

660

661

662

663

664

665

666

667

668

669

670

671

672

673

674

675

676

677

678

679

680

681

682

683

684

685

686

687

688

689

690

691

692

693

694

695

696

697

Cassandra servers in the cluster and created enough fake connections, they could drive it out of service (Nayak \& Poriya 2013).

- Inactive connections: Cassandra does not set the timeout value for inactive connections; consequently, it has a problem in managing inactive connections (Nayak, 2013; Paterson, 2006).

\subsubsection{DynamoDB}

- Introduction:

DynamoDB is a Closed source NoSQL DBMS used by Amazon. Its initial release appeared in January 2012 (Grolinger \& Higashino, 2013). DynamoDB was developed using Java (Meier \& Kaufmann, 2019). It is fast and flexible and supports both the document and key-value data models (Paterson, 2006). The query language is proprietary in DynamoDB but MapReduce and REST query are supported (Grolinger \& Higashino, 2013).

- Security Analysis:

- Authentication: Authentication is supported by DynamoDB (Paterson, 2006). It is ensured by the integration of Identity and Access Management (IAM services (Grolinger, 2013; Paterson, 2006).

- Authorization: Authorization is supported by DynamoDB (Paterson, 2006). It allows users to create policies and operations on domains (Grolinger \& Higashino 2013). - Auditing: DynamoDB integrates with Amazon Cloud Watch Service. Access information about latencies for operations, requests throughput, and the amount of data stored (Grolinger \& Higashino 2013) are subject to auditing at the ACWS.

- Data Encryption: DynamoDB does not support the encryption of data at rest (Grolinger \& Higashino, 2013). Communication between the client and the server uses the https protocol (Paterson, 2006).

\section{QUERY LANGUAGE}

A query language is a set of user-entered statements that define, manipulate, and retrieve data from database and information systems by sending queries. NoSQL DBMSs don't use a standard query language, and most of their providers have developed their query languages. This brings some stumbling blocks when users want to switch from one NoSQL DBMS to another; for instance, Cassandra supports Cassandra Query Language (CQL), while MongoDB uses Mongo query language, and so on. To resolve this, a common query language that can support a variety of NoSQL database users was needed. Unstructured Query Language, or UnQL (pronounced 'uncle'), is a joint effort of several NoSQL DBMS providers that brings a commonplace and standardized data definition, manipulation, and retrieval language to the NoSQL platform. UnQL is being developed by the creators of Couch and SQLlite. It is considered as the superset of SQL; providing a SQL-like syntax, and familiarity to the database developers and users. The model and syntax of the UnQ1 is appropriate for the unstructured, self-describing data formats. UnQ1 provides features to fetch and process complex document structures. It also provides the elasticity of the NoSQL schema-less design and the structured table format of the relational database. Data stored in JSON format as well as in document and non-relational stores can be queried using UnQ1. UnQ1 is open for developers and academic communities for further enhancements and development (Nayak \& Poriya, 2013).

PeerJ Comput. Sci. reviewing PDF | (CS-2020:04:48359:2:0:NEW 24 Jul 2020) 
698

699

700

701

702

703

704

705

706

707

708

709

710

711

712

713

714

715

716

717

718

719

720

721

722

723

724

725

726

727

728

729

730

731

732

733

734

735

736

737

738

739

\section{COMPARISON OF NOSQL DATABASES CATEGORIES}

A generic introduction to NoSQL databases through the categorization of different NoSQL databases is presented in Table 1 (Strauch \& Sites, 2011). If we add the Object Store NoSQL systems to the table, it becomes as follows:

\section{SUMMARIZED TABLE}

The aforementioned exhaustive study is summarized in Table 2, which presents the result of the comparison between the mentioned DBMSs in terms of the selected determining criteria.

\section{NoSQL DBMS SELECTION ALGORITHM}

In this section, we propose a smart agent algorithm which selects NoSQL DBMSs according to the user's needs - in our case, that would be selecting the most convenient DBMS for large medical records storage. The algorithm gives a weight to each criterion, measured as (user weight/total weights). The user sets the appropriate weights based on their needs. The algorithm arranges weights in an ascending order, and returns an ordered table according to the arranged weights.

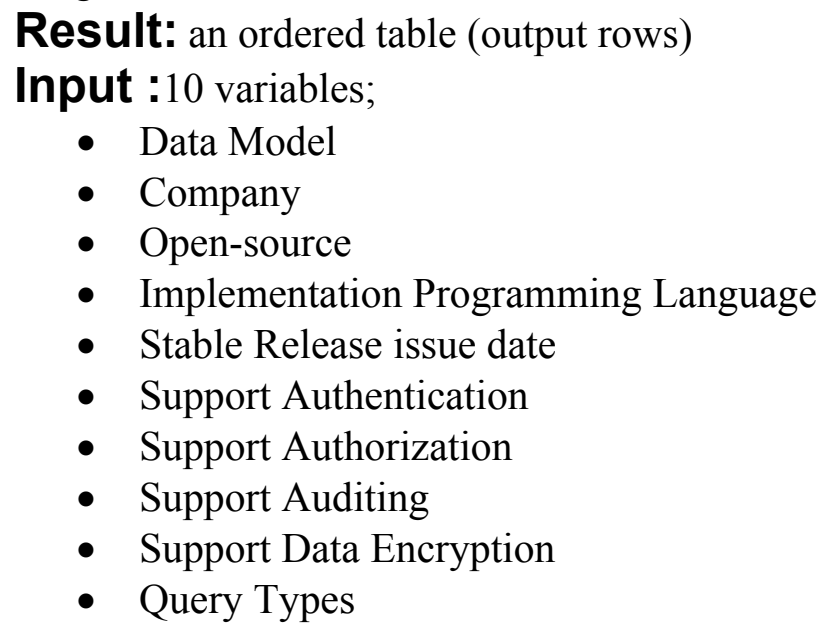


740

741

742

743

744

745

746

747

748

749

750

751

752

753

754

755

756

757

758

759

760

761

762

763

764

765

766

767

768

769

770

771

772

773

774

775

776

777

778

779

780

if all input! = null then

sort inputs incrementally according to their weights.

\section{end}

arranged weight list $=$ arrange (Inputs Weight).

${ }^{*}$ Extract all tuples from the table that satisfy the user's constraints

and store data in the selected list. */

selected List $=$ Select $*$ from table where column name equal inputs value for each given input value.

output rows =sort selected List function (the maximum value from arrange weight list, selected list)

\section{Algorithm: NoSQL DBMS selection algorithm}

\section{Results and Discussion}

LDAP is a directory service and a searchable database repository that allows authorized users and services to find information related to people, computers, network devices, and applications (Lasisi \& Ajagbe, 2012). LDAP directories are used mostly for reads, and its servers are simple to install and maintain. LDAP is an Open Standard Protocol and is Lightweight. Moreover, it offers common access for multiple database management systems such that MongoDB, MySQL, PostgreSQL, Oracle 9i, IBM DB2, etc.

Apache Directory Studio is an integrated directory tool platform intended for use with any LDAP server, but is specifically designed to be used with ApacheDS. Creating and launching a new LDAP server now takes less than 10 seconds (Apache Software Foundation, 2020).

JSON Web Token (JWT) is an open standard that characterizes a minimized and independent approach to safely move data between parties as a JSON object. (IETF JWT, 2020).

Spring Boot gives a good platform to Java designers to build up an independent and productiongrade spring application that you can simply run (Pivotal Team, 2020).

Logback is expected as a replacement to the famous $\log 4 \mathrm{j}$ venture, picking up where $\log 4 \mathrm{j} \operatorname{leaves}$ off.

This paper presents a set of functions, based on web services. The latter offers a set of endpoints that include authentication, authorization, auditing, and encryption of information. It will be secure to use the client created via these web services in all types of NoSQL DBMS.

This section will explain the web services identified.

First, web services related to the authentication process depend on authentication through the Apache directory server. In this process, the users and units they belong to are defined within the Apache server, so that the username and password are sent via secure channel. If the user credentials are valid, JSON Web Token (JWT) returns all the user's information including their role within the organization and the units they belong to. With the returned information, NoSQL DB allows the user to log into the database after ensuring that the user is authorized. If the validation process fails, access is denied. This web service related to the authentication process not only allows for user verification, but also provides all the processes related to the user management, such as creating a new user, updating, or deleting an existing user within the organization. The returned information might be useful for databases in one way or another. It 
781

782

783

784

785

786

787

788

789

790

791

792

793

794

795

796

797

798

799

800

801

802

803

804

805

806

807

808

809

810

811

812

813

814

815

816

817

818

819

also facilitates the interaction with the authentication server. The authentication process is done by the authentication controller, which contains two main functions: the first is the generate JWT token function, which is responsible for completing the user authentication process and returning the user's token. The second is responsible for validating the token, and ensuring that it is valid for use and its content has not been changed or that its validity has not expired.

The other user-related processes in the authentication server are performed by the user CRUD Controller. This controller contains five main functions, which can be described as follows:

- Get all users: which gets all information to of all users on the authentication server and returns JWT token with the essential information, such as display name, user name, organizational unit, ... etc.

- Get user by UID: which returns specific user's information by the UID.

- Delete user by UID: which deletes any user by the UID.

- Create a new user: which creates a new user within the organization.

- Update User by UID: which updates the information of any user by UID inside the authentication server.

All processes related to the authentication processes are shown in Fig. 2. and Fig. 3.

Second, the web services related to the authorization process. Most of the processes related to authorization are built within the databases. in this case, authentication is done from outside, and the user's predefined privileges are used within the databases. But sometimes the authorized operations may be related to the roles of people within the organization, so that the databases enable users to perform the operations they are allowed to. We hereby provide a solution to authorize the usage of the role within the organization, so that they are allowed/rejected to perform their operations through their organizational units. Databases can thus allow/reject users' operations based on their roles in the organization. The authorization process is done using an authorization controller that performs five basic functions. They can be described as follows:

- Get all groups (organization unit): By using this function within the NoSQL DB, the whole organization unit inside the server is returned. The returned values contain important information such as organization unit (OU) name and description.

- Get a group by OU name: By using this function inside the NoSQL DB, OU information is returned by the OU name.

- Delete the group by OU name.

- Create a new OU: This function enables the NoSQL DB to create a new group within the organization.

- Update group by OU name: This function enables updating any OU information within the apache directory server.

All processes related to the authorization processes are shown in Fig. 4.

Third, web services related to the auditing process. the auditing process is crucial for any type of database. Auditing documents all the operations that occur in the databases. We offer a set of APIs related to the auditing process, based on the Logback. The proposed solution contains the 
820 auditing controller, which contains a basic function to define the auditing process. Here is either 821 the INFO, ERROR or WARN and the content to be stored in the auditing file. The databases will 822 thus be able to easily record all the processes that have been performed along with their specific 823 timestamp. Fig. 5. shows one such recorded audit operations.

824 Fourth, web services related to the encryption process. Since securing such sensitive data as 825 medical records is absolutely critical on $\mathrm{DB}$, encryption is a basic requirement within NoSQL 826 DB. Encryption helps to store the information safely and confidentially so that it is not being 827 accessed by unauthorized users. This paper offers a set of web services to encrypt sensitive data, 828 such as RSA, Triple DES, AES, and BlowFish. The web services contain four types of 829 controllers; namely, AESController, BlowfishSsecretKey, TDESController, and RSAController. 830 Each controller includes two functions. One is used for encryption, the other for decryption; with

831 the possibility of storing the used keys easily and changing them if necessary. Consequently, 832 NoSQL DB can use one of the four strongest encryption cryptosystems to encrypt sensitive data. 833 All processes related to the encryption processes are shown in Fig. 6.

834 The APIs described in the above paragraphs are illustrated in Fig. 7.

835 This figure shows the integration between the proposed system and NoSQL DBMS.

836

- Login request: In this request, the user logs in to the NoSQL DBMS. NoSQL DBMS

837

838 makes a login request to the Apache directory server with the password and username

839 sent. The Apache directory server checks the username and password and returns a JWT token in case of success; access is denied in case of failure. The JWT token is sent with

840

841

842

843

844

845

846

847

848

849 all requests made to the proposed system.

- Authorization request: The user makes a query that requires verification of its privileges. The NoSQL DBMS checks the user's permissions, either from the stored JWT token, or the Apache directory server. NoSQL DBMS completes or rejects the query based on the person's authorization.

- Auditing request: The NoSQL DBMS uses the audit API to record all operations performed on it. The DB admin obtains the audit report to review all operations that occurred on the NoSQL DBMS.

850

- Encryption/Decryption request: The NoSQL DBMS uses the API to encrypt and decrypt all sensitive information using four different types of encryption algorithms.

It is important to mention that in the testing phase, all communications between NoSQL DB and web services will be secured with HTTPS instead of HTTP, for the data to be transmitted in a protected channel using a self-signed certificate. In the live phase, using an authentic certificate from a certificate authority is recommended.

\section{Contribution:}

\section{This paper presented:}

857

1. A taxonomy of NoSQL systems categories and their DBMSs.

2. A comparative study among NoSQL DBMS, along with several criteria including security issues. 
859

860

861

862

863

864

865

866

867

868

869

870

871

872

873

874

875

876

877

878

879

880

881

882

883

884

885

886

887

888

889

890

891

892

893

894

895

896

3. An algorithm to select the most convenient NoSQL DBMS for a given application. For our current purposes, this algorithm can be used to select the appropriate solution for the COVID-19 patients', medical staff, and organizations data. An API based security system. It is DBMS independent, and may be used to embed security in any NoSQL DBMS. This achieves the goal of securing COVID-19 related data.

\section{Conclusions}

This research paves the way for the use of NoSQL databases to store and protect COVID-19 patients' information, removing existing hurdles to their adoption. It does so by understanding the five main categories of NoSQL databases, offering a comparative study among these categories based upon a set of comparison criteria, namely Performance, Scalability, Flexibility, Complexity, Functionality, and Security issues. This comparative study reveals the strengths and weaknesses of each category. The most commonly used database management systems in each category were discussed. DBMSs were discussed from two points of view: the pertained to the information, the second the security analysis. Building on that discussion, a table was built to compare the strengths and weaknesses of each DBMS, according to their comparison criteria are Data Model, Company, Open source, Implementation Programming Language, Stable Release issue dates, Supporting Authentication, Support Authorization, Support Auditing, Support Data Encryption, and Query Types. The table provides individuals users and organizations a clear understanding of the various NoSQL DBMSs. The choice of a NoSQL DBMS will depend on its convenience to store and secure the information of COVID-19 patients. For this purpose, a new decision-support algorithm was developed, to assist in the selection process of the most secure and convenient NoSQL DBMS to store and maintain the security of the information collected on each individual patient.

The paper developed and presented a complete solution to the essential security problems common to all types of NoSQL DBMSs, thus successfully resolving their weaknesses, which previously kept them from being the favored storage solution for sensitive data. Those solutions relate to the processes of authentication, authorization, auditing, and encryption of sensitive information. Moreover, the proposed solution is sufficiently general to allow the patients' database designer to freely choose any NoSQL DBMS to implement their design, while successfully resolving those security pitfalls.

\section{References}

Abed Amira Hassan. (2020). "Recovery and Concurrency Challenging in Big Data and NoSQL Database Systems." 11(04): 4321-4329.

Angles Renzo. 2012. A comparison of current graph database models. In Data Engineering Workshops (ICDEW), 2012 IEEE 28th International Conference on. IEEE, 171-177. Apache Software Foundation (2020). Available at https://directory.apache.org/studio/ (accessed 24 April 2020). 
897 Arora Rupali and Rinkle Rani Aggarwal. 2013. An Algorithm for Transformation of Data 898 from MySQL to NoSQL (MongoDB). International Journal of Advanced Studies in Computer 899 Science and Engineering 2, 1 (2013), 6-12.

900 Chen, Min, Shiwen Mao, and Yunhao Liu."Big data: A survey." Mobile networks and 901 applications (2014) 19(2): 171-209.

902 Chodorow, Kristina. MongoDB: the definitive guide: powerful and scalable data storage, " 903 O'Reilly Media, Inc."(2013).1-432 pages.

904 Chang, Fay, J. Dean, S. Ghemawat, W. C. Hsieh, D. A. Wallach, M. Burrows, T. Chandra, 905 A. Fikes and R. E. Gruber (2008). "Bigtable: A distributed storage system for structured data." 906 ACM Transactions on Computer Systems (TOCS) 26(2): 4.

907 Dayan Niv, Manos Athanassoulis, and Stratos Idreos. 2018. Optimal Bloom Filters and 908 Adaptive Merging for LSM-Trees. ACM Trans. Database Syst. 43, 4, Article 16 (Dec. 2018), 48 909 pages. https://doi.org/10.1145/3276980.

910 Deka Ganesh Chandra. A survey of cloud database systems. IT Professional 16(2) (2014), 5091157.

912 Dindoliwala, Vaishali. J. and R. D. Morena (2017). "Survey on Security Mechanisms In 913 NoSQL Databases." International Journal of Advanced Research in Computer Science 8(5).

914 Elmasri, Ramez. and S. Navathe (2017). Fundamentals of database systems, Pearson.

915 Franz Inc.(2020). AllegroGraph. http://www.franz.com/agraph/allegrograph.2020 (accessed 916 April 23, 2020)

917 George, S. (2013). "NoSQL-NOT ONLY SQL." International Journal of Enterprise Computing 918 and Business Systems 2: 11.

919 Grolinger, Katarina, Wilson A Higashino, Abhinav Tiwari, Miriam AM Capretz, (2013).

920 "Data management in cloud environments: NoSQL and NewSQL data stores." 2(1): 22.

921 Han, J., E. Haihong, G. Le and J. Du (2011). Survey on NoSQL database. 2011 6th

922

923

924

925 international conference on pervasive computing and applications, IEEE.

Jim Trevor, Nikhil Swamy, and Michael Hicks. 2007. Defeating script injection attacks with browser-enforced embedded policies. In Proceedings of the 16th international conference on World Wide Web. ACM, 601-610.

926 IETF JWT 2020. Available at https://jwt.io/introduction/ (accessed 24 April 2020).

927 Kaur, K. and R. Rani (2013). Modeling and querying data in NoSQL databases. 2013 IEEE 928 International Conference on Big Data, IEEE.

929 Khasawneh Tariq, Mahmoud AL-Sahlee, Ali Safia (2020). SQL, NewSQL, and NOSQL 930 Databases: A Comparative Survey (2020). 11th International Conference on Information and 931 Communication Systems (ICICS), IEEE.

932 Kobrix Software Inc. (2020). The HyperGraphDB. www.hypergraphdb.org.2020(accessed 933 April 23, 2020).

934 Lakshman, A. and P. Malik (2010). "Cassandra: a decentralized structured storage system." ACM SIGOPS Operating Systems Review 44(2): 35-40.

Lasisi, Ayodele Nojeem, Musibau Akintunde Ajagbe. Communications (2012). "Samba OPENLDAP: An Evolution And Insight." 2(3): 354-362

938 Lourenço, J. R., B. Cabral, P. Carreiro, M. Vieira and J. Bernardino (2015). "Choosing the 939 right NoSQL database for the job: a quality attribute evaluation." Journal of Big Data 2(1): 18.

940 Meier, A. and M. Kaufmann (2019). SQL \& NoSQL Databases, Springer. 
941 Moniruzzaman, A. B. M. and S. A. Hossain (2013). "NoSQL Database: New Era of Databases 942 for Big data Analytics - Classification, Characteristics and Comparison." CoRR abs/1307.0191. 943 Nayak, A., A. Poriya and D. Poojary (2013). "Type of NOSQL databases and its comparison 944 with relational databases." International Journal of Applied Information Systems 5(4): 16-19.

945 Noiumkar Preecha and Tawatchai Chomsiri. 2014. A comparison the level of security on top

9465 open source NoSQL databases. In The 9th International Conference on Information

947 Technology and Applications (ICITA2014). 6.

948 Özsu, M.T. and Valduriez, P., (2020). NoSQL, NewSQL, and Polystores. In Principles of

949 Distributed Database Systems (pp. 519-557). Springer, Cham.

950 Pivotal Team 2020. Available at https://spring.io/projects/spring-boot (accessed 24 April 2020).

951 Paterson Jim, Stefan Edlich, Henrik Hrning, and Reidar Hrning. 2006. The Definitive

952 Guide to db4o. Springer. 485 pages.

953 Sahafizadeh, E. and M. A. Nematbakhsh (2015). "A survey on security issues in Big Data and

954 NoSQL." Advances in Computer Science: an International Journal 4(4): 68-72.

955 SecurityFocus (2020). Available at https://www.securityfocus.com/bid/66474/discuss

956 (accessed 27 April 2020).

957 Son Sooel, Kathryn S McKinley, and Vitaly Shmatikov. 2013. Diglossia: detecting code

958 injection attacks with precision and efficiency. In Proceedings of the 2013 ACM SIGSAC

959 conference on Computer \& communications security. ACM, 1181-1192.

960 Strauch, C., U.-L. S. Sites and W. Kriha (2011). "NoSQL databases." Lecture Notes, Stuttgart

961 Media University 20.

962 Sullivan Bryan, and Vincent Liu. 2011. Web application security, a beginner's guide (1 ed.).

963 McGraw-Hill Education Group. 1-352 pages.

964 Tudorica, B. G. and C. Bucur (2011). A comparison between several NoSQL databases with

965 comments and notes. 2011 RoEduNet international conference 10th edition: Networking in

966 education and research, IEEE.

967 Zahid, A., R. Masood and M. A. Shibli (2014). Security of sharded NoSQL databases: A

968 comparative analysis. 2014 Conference on Information Assurance and Cyber Security (CIACS),

969 IEEE.

970 Zaki Asadulla Khan. 2014. NoSQL databases: new millennium database for big data, big users,

971 cloud computing and its security challenges. International Journal of Research in Engineering

972 and Technology (IJRET) 3, 15 (2014), 403-409.

973 Zugaj, Wilhelm, and Anita Stefanie Beichler. (2019). "Analysis of Standard Security Features

974 for Selected NoSQL Systems." 3(2): 41-49.

975 


\section{Table $\mathbf{1}$ (on next page)}

Comparison of NoSQL databases categories 
Table 1 comparison of NOSQL databases categories

\begin{tabular}{|c|c|c|c|c|c|}
\hline $\begin{array}{c}\text { NoSQL DB } \\
\text { Categories }\end{array}$ & Performance & Scalability & Flexibility & Complexity & Functionality \\
\hline $\begin{array}{c}\text { Document } \\
\text { stores }\end{array}$ & High & $\begin{array}{c}\text { Variable } \\
\text { (High) }\end{array}$ & High & Low & Variable (Low) \\
\hline $\begin{array}{c}\text { Key-Value } \\
\text { Stores }\end{array}$ & High & High & High & None & Variable (None) \\
\hline $\begin{array}{c}\text { Graph DB } \\
\text { Column stores }\end{array}$ & High & High & Moderate & High & Graph Theory \\
\hline Object Store & High & $\begin{array}{c}\text { Variable } \\
\text { (High) }\end{array}$ & High & Low & $\begin{array}{c}\text { Object Oriented } \\
\text { Programming }\end{array}$ \\
\hline
\end{tabular}

3 


\section{Table 2 (on next page)}

Summarized Table 


\begin{tabular}{|c|c|c|c|c|c|c|c|c|c|c|}
\hline 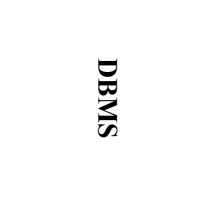 & $\frac{3}{0}$ & $\stackrel{\varrho}{\stackrel{E}{E}}$ & 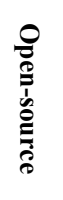 & 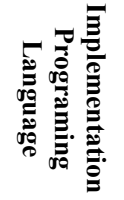 & 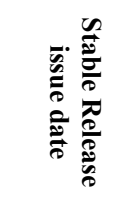 & 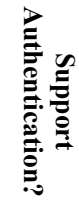 & 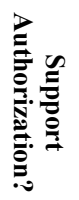 & 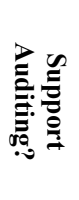 & 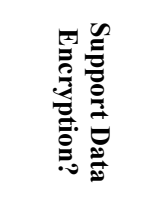 & 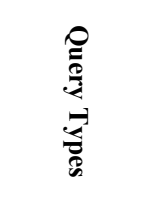 \\
\hline MongoDB & Document & $\begin{array}{l}\text { MongoDB } \\
\text { Inc. }\end{array}$ & Yes & $\begin{array}{l}\mathrm{C}++, \mathrm{C}, \\
\text { JavaScrip }\end{array}$ & June 2018 & Yes & Yes & weak & $\begin{array}{l}\text { Yes, but not } \\
\text { support data } \\
\text { at rest } \\
\text { encryption }\end{array}$ & $\begin{array}{l}\text { Mongo } \\
\text { Query } \\
\text { Language, } \\
\text { REST, } \\
\text { MapReduce }\end{array}$ \\
\hline CouchDB & Document & apache & Yes & Erlang & $\begin{array}{l}\text { November } \\
2017\end{array}$ & Yes & Yes & weak & $\begin{array}{l}\text { No, only } \\
\text { Client/Server } \\
\text { SSL-based }\end{array}$ & $\begin{array}{l}\text { Map reduce, } \\
\text { REST }\end{array}$ \\
\hline $\begin{array}{c}\text { Couchbase } \\
\text { Server }\end{array}$ & Document & $\begin{array}{l}\text { Couchbase } \\
\text { Inc. }\end{array}$ & Yes & $\begin{array}{l}\text { Erlang, } \\
\text { C, C++ }\end{array}$ & $\begin{array}{l}\text { February, } \\
2018\end{array}$ & Yes & No & No & No & $\begin{array}{l}\text { SQL++, } \\
\text { Map reduce, } \\
\text { REST }\end{array}$ \\
\hline Redis & Key-Value & $\begin{array}{l}\text { Salvatore } \\
\text { Sanfilippo }\end{array}$ & Yes & $\mathrm{C}, \mathrm{C}++$ & June ,2018 & weak & No & No & No & $\begin{array}{l}\text { REST by } \\
\text { Third-party } \\
\text { APIs }\end{array}$ \\
\hline Voldemort & Key-Value & LinkedIn & Yes & Java & July ,2017 & No & No & No & No & Map reduce \\
\hline $\begin{array}{c}\text { Oracle } \\
\text { BerkeleyDB }\end{array}$ & Key-Value & Oracle & No & $\begin{array}{l}\mathrm{C}, \mathrm{C}++, \\
\text { Java }\end{array}$ & June ,2018 & No & No & No & $\begin{array}{l}\text { No, only } \\
\text { data at rest }\end{array}$ & $\begin{array}{l}\text { SQLite, } \\
\text { REST }\end{array}$ \\
\hline Neo4j & Graph & $\begin{array}{l}\text { Neo } \\
\text { Technology }\end{array}$ & Yes & Java & $\begin{array}{l}\text { April } \\
\text {,2018 }\end{array}$ & No & No & No & $\begin{array}{l}\text { No, only } \\
\text { Client/Server } \\
\text { SSL-based }\end{array}$ & $\begin{array}{l}\text { Cypher, } \\
\text { Gremlin and } \\
\text { SparQL, } \\
\text { REST }\end{array}$ \\
\hline HyperGraphDB & Graph & $\begin{array}{l}\text { Kobrix } \\
\text { Software, } \\
\text { Inc. }\end{array}$ & Yes & Java & May, 2017 & No & No & No & No & $\begin{array}{l}\text { SQL like } \\
\text { querying, } \\
\text { REST }\end{array}$ \\
\hline AllegroGraph & Graph & Franz Inc. & No & $\begin{array}{l}\text { Common } \\
\text { Lisp }\end{array}$ & $\begin{array}{l}\text { October, } \\
2017\end{array}$ & Yes & Yes & weak & $\begin{array}{l}\text { No, only } \\
\text { Client/Server } \\
\text { HTTPS }\end{array}$ & $\begin{array}{l}\text { SparQL and } \\
\text { Prolog, } \\
\text { REST }\end{array}$ \\
\hline HyperTable & Column-based & $\begin{array}{l}\text { Zvents } \\
\text { before }\end{array}$ & Yes & $\mathrm{C}++$ & $\begin{array}{l}\text { March } \\
, 2016\end{array}$ & No & No & No & No & HQL \\
\hline HBase & Column-based & apache & Yes & Java & April,2018 & Yes & Yes & Yes & $\begin{array}{l}\text { No, only } \\
\text { Client/Server }\end{array}$ & $\begin{array}{l}\text { Map reduce, } \\
\text { REST }\end{array}$ \\
\hline GemStone & Object Store & GemTalk & Yes & Smalltalk & June, 2018 & Yes & Yes & weak & No & $\begin{array}{l}\text { No querying } \\
\text { like SQL }\end{array}$ \\
\hline db4o & Object Store & Actian & Yes & Java, C\# & Sep. ,2011 & weak & Yes & No & weak & db4o-sql \\
\hline Objectivity/DB & Object Store & $\begin{array}{l}\text { Objectivity } \\
\text { Inc }\end{array}$ & No & $\begin{array}{l}\text { Java, C\#, } \\
\text { C++ and } \\
\text { Paython }\end{array}$ & June 2017 & Yes & weak & No & No & OQL \\
\hline Cassandra & Multi-model & apache & Yes & Java & $\begin{array}{l}\text { February, } \\
2018\end{array}$ & Yes & Yes & weak & Yes & $\begin{array}{l}\text { CQL, Map } \\
\text { reduce, } \\
\text { REST by } \\
\text { Third-party } \\
\text { APIs }\end{array}$ \\
\hline DynamoDB & Multi-model & Amazon & No & Java & 2012 & No & No & weak & No & $\begin{array}{l}\text { Map reduce } \\
\text { REST }\end{array}$ \\
\hline
\end{tabular}


Figure 1

\section{NoSQL Database Management Systems Classification}

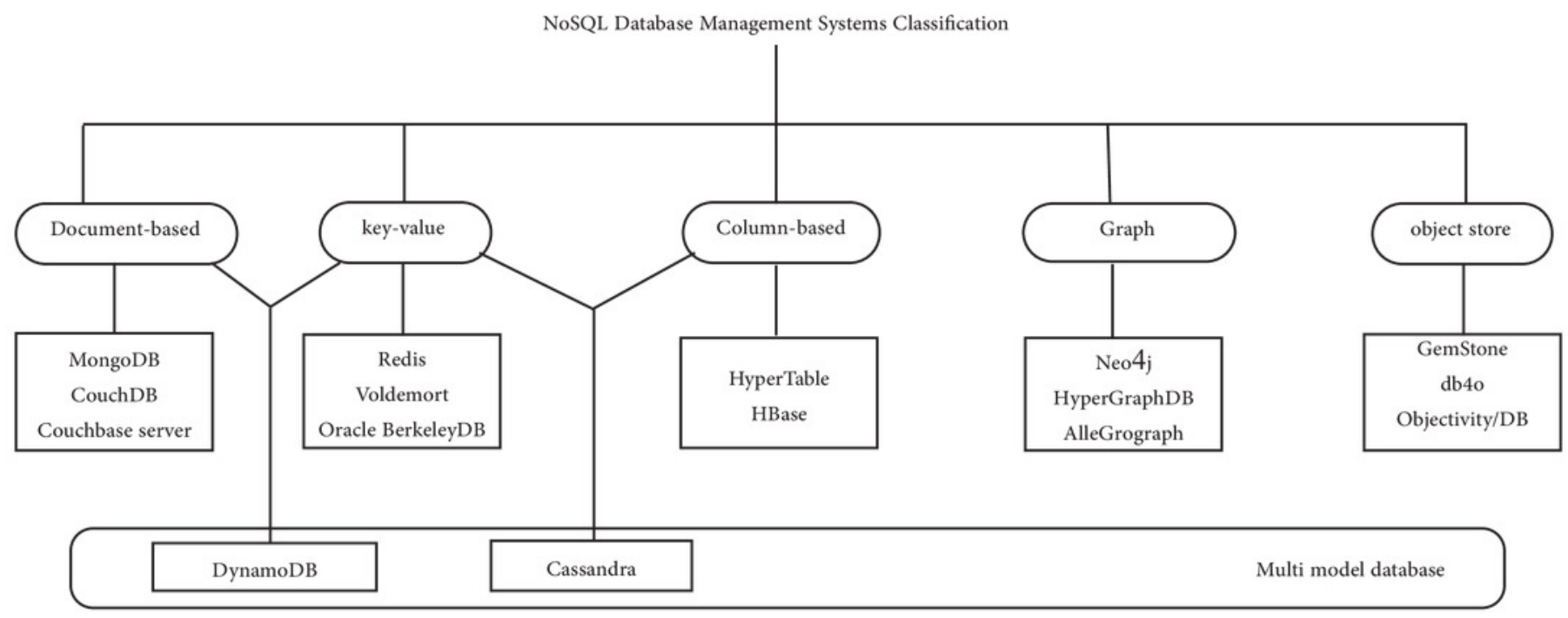




\section{Figure 2}

\section{Authentication processes (a)}
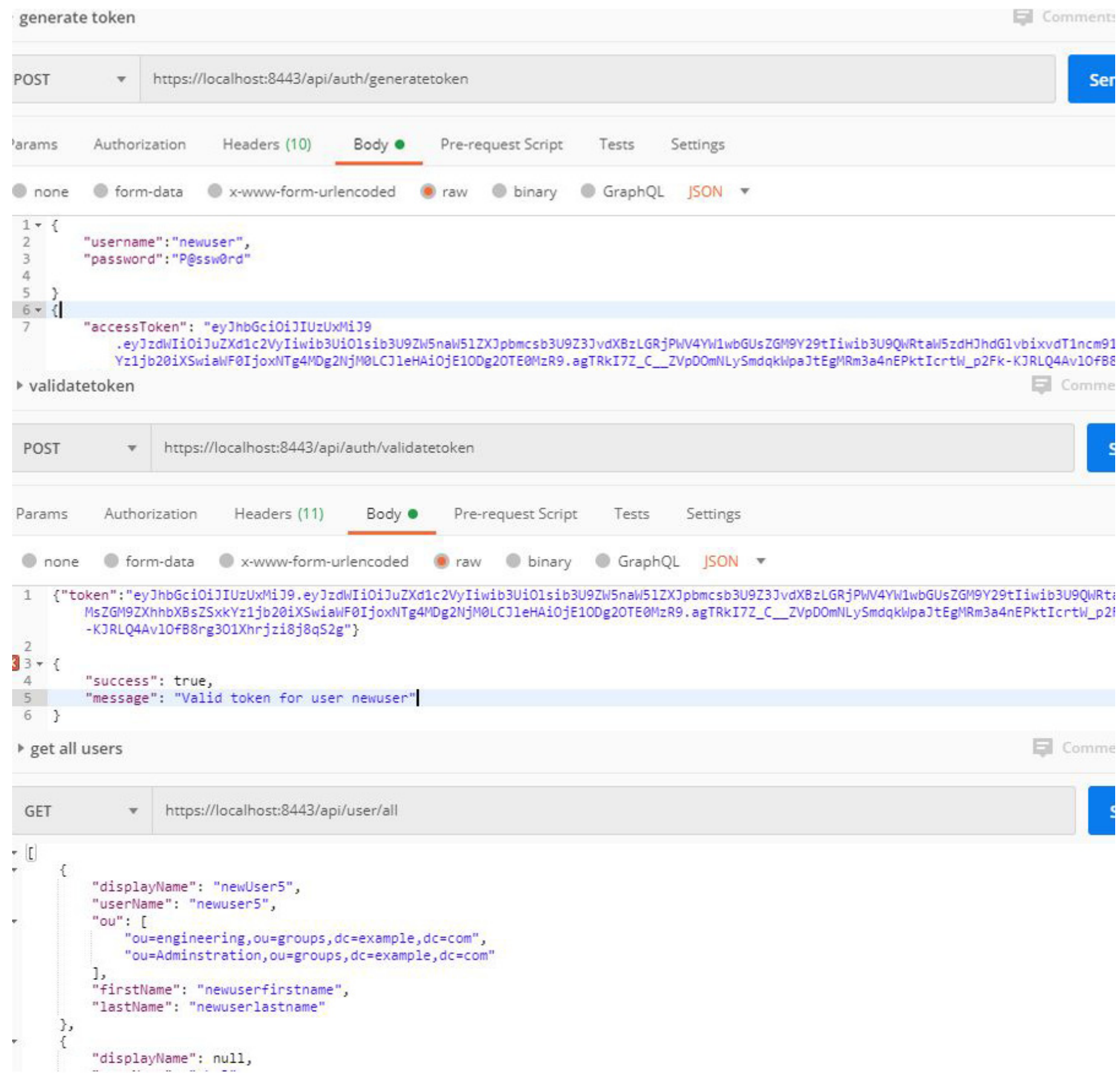

DELETE v https://localhost:8443/api/user/newuser2

1 User newuser 2 is deleted successfully 
Figure 3

Authentication processes (b) 


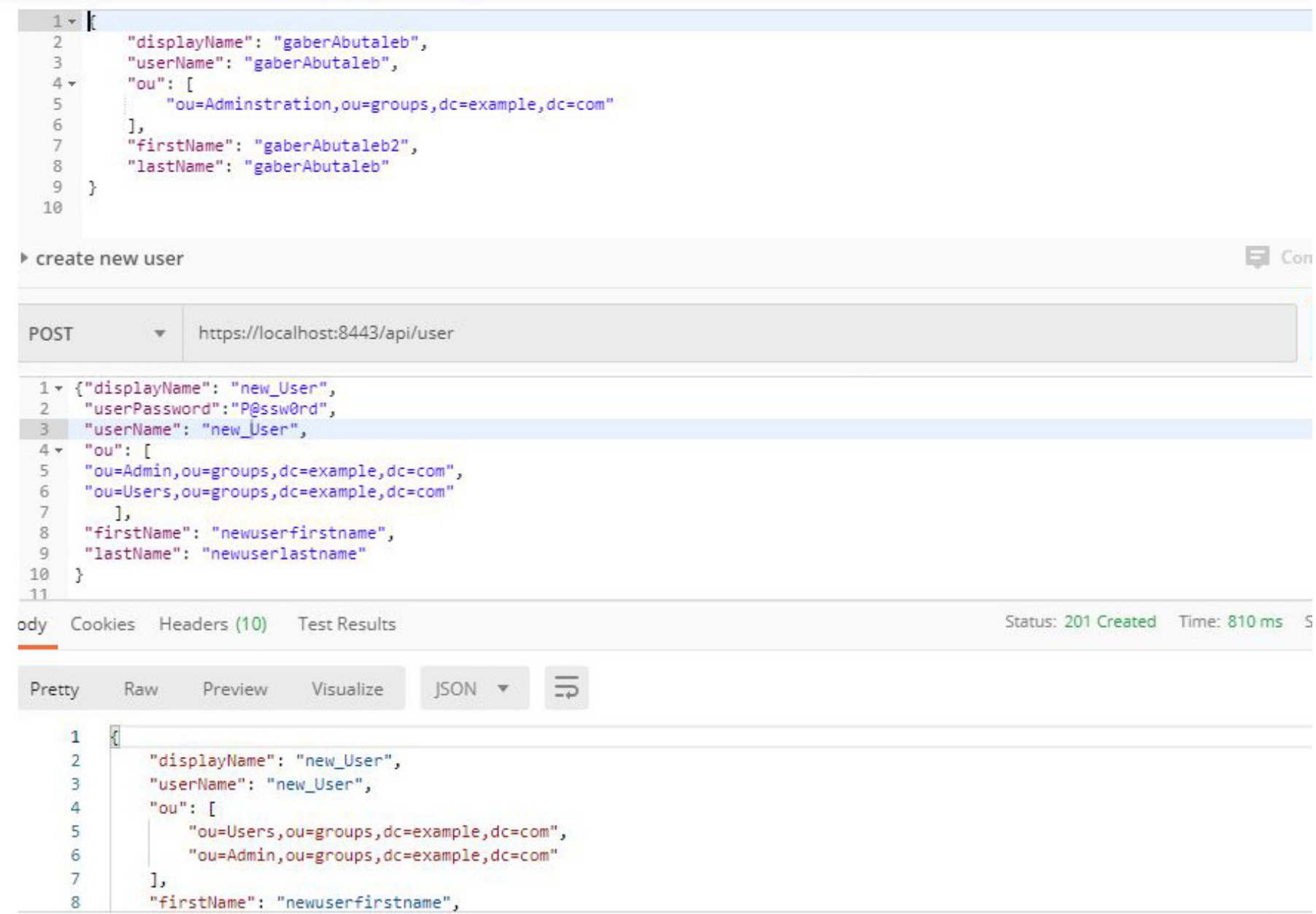

- update user

\section{PUT $\quad$ https://localhost:8443/api/user/gaberAbutaleb}

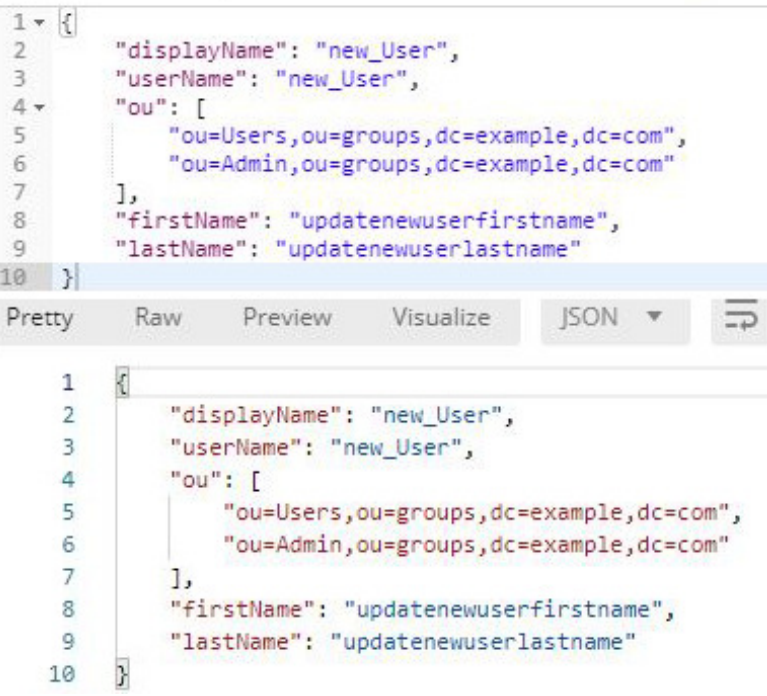


Figure 4

Authorization processes 


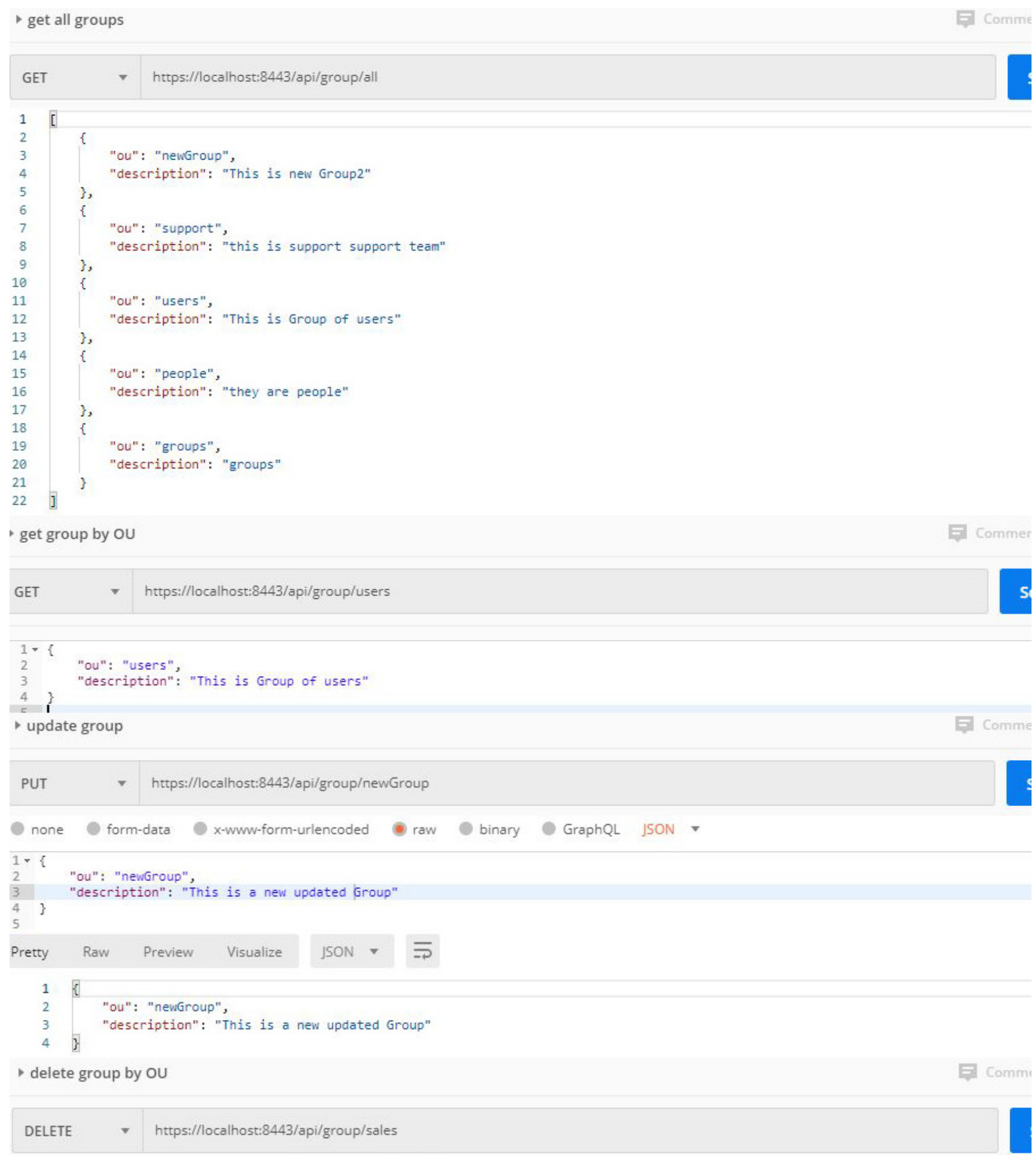

1 Group is deleted Successfully 


\section{Figure 5}

\section{Auditing process}

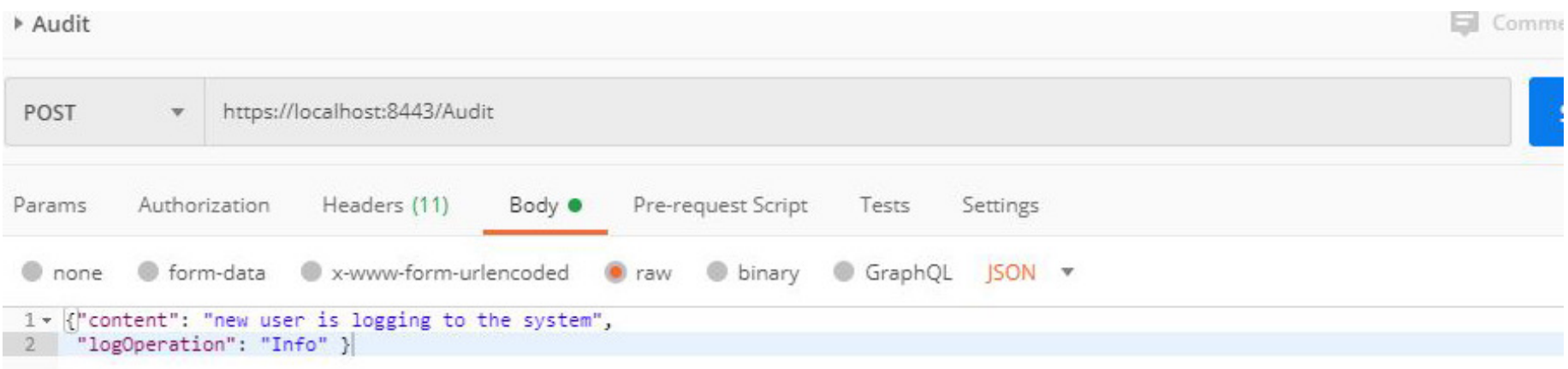

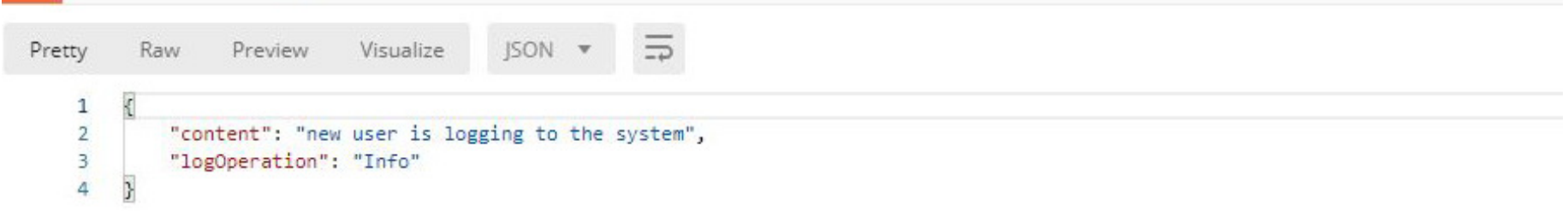

2020-04-28 17:49:22.646 ERROR 15348 --- [https-jsse-nio-8443-exec-7] com.nosql.controller. UserController Error when login user newuser try to delte user with uid newuser 5

2020-04-28 17:50:16.658 INFO 15348--- [https-jsse-nio-8443-exec-9] com.nosql.controller.UserController : login user newuser create new user with uid new User

2020-04-28 17:58:14.398 INFO 15348 --- [https-jsse-nio-8443-exec-3] com.nosql.controller.UserController : login user newuser update new with uid gaberAbutaleb

2020-04-28 18:12:16.561 WARN 15348--- [https-jsse-nio-8443-exec-5] o.s.ldap.odm.core.impl.ObjectMetaData : The Entry class Group should be declared final

2020-04-28 18:12:16.643 INFO 15348 --- [https-jsse-nio-8443-exec-5] com.nosql.controller.GroupController : Login user newuser get all Group

2020-04-28 18:17:14.889 INFO 15348 --- [https-jsse-nio-8443-exec-7] com.nosql.controller.GroupController : login user newuser get Group with ou users

2020-04-28 18:20:03.839 INFO 15348 --- [https-jsse-nio-8443-exec-2] com.nosql.controller.GroupController : login user newuser update new group with ou newGroup

2020-04-28 18:23:14.112 ERROR 15348 --- [https-jsse-nio-8443-exec-9] com.nosql.controller.GroupController Error when login user newuser try to delte Group with ou sales

2020-04-28 19:03:14.271 ERROR 15348 --- [https-jsse-nio-8443-exec-3] c.n.s.JwtAuthenticationEntryPoint Responding with unauthorized error. Message - Full authentication is required to access this resource 2020-04-28 19:03:24.800 INFO 15348 --- [https-jsse-nio-8443-exec-8] com.nosql.Auditing.Audit new user is logging to the system

2020-04-28 19:10:32.979 ERROR 15348 --- [https-jsse-nio-8443-exec-5] com.nosql.Auditing.Audit Error while call the system

2020-04-28 19:12:15.107 WARN 15348 --- [https-jsse-nio-8443-exec-9] com.nosql.Auditing.Audit 
Figure 6

\title{
Encryption processes
}

\author{
- TDES ENCRYPT \\ POST $\quad$ https://localhost:8443/TDESencrypt \\ Params Authorization Headers (10) Body Pre-request Script Tests Settings \\ none form-data $\bigcirc$-www-form-urlencoded raw binary GraphQL Text \\ 1 Welcome in Tdes Encryption \\ Body Cookies Headers (10) Test Results \\ Pretty Raw Preview Visualize Text $>\equiv$ \\ 1 SPPRYaouX37/89RrhXvQHJS5hlfzipqa/4J5VxrpYyQ= \\ - TDES DECRYPT \\ POST $\quad$ https://localhost:8443/TDESdecrypt

\begin{tabular}{|c|c|c|c|c|c|c|}
\hline Params & Authorization & Headers (10) & Body & Pre-request Script & Tests & Settings \\
\hline
\end{tabular} \\ 1 sPPRYaouX37/89RrhXvQHJS5hlfzipqa/4J5VxrpYyQ= \\ 2 \\ Body Cookies Headers (10) Test Results \\ Status: $200 \mathrm{OK}$ Time: $171 \mathrm{~ms}$

\begin{tabular}{cl|c|c|} 
Pretty Raw Preview Visualize & Text \\
1 & Welcome in Tdes Encryption
\end{tabular}

\section{POST \\ v https://localhost:8443/encrypt} \\ Welcome in RSA \\ FFCsOyta@0aKUo1oNmLdZCEHWgquvDyPj \\ 1C9GrtAOCUZd fCwLBUUQiuqAZdJ IuD8KAAYt \\ 4+heZuQI3YrX7EBWlkXAODvIrSOseR891pqWw \\ ulMfbvVi+BXnkIGaholkMzAQKrLkqoSuNW3 z 9 \\ GXkd5+5riKEqkYRUehKqv9ygFovW7g= \\ POST V https://localhost:8443/dencrypt \\ FFCsOyta@OaKUo10NmLdZCEHWgquvDyPj \\ 1C9GrtAOCUZdfCwLBUUQiuqAZdJ luD8KAAYt \\ 4+heZUQI3YrX7EBWkXAODvIrSOseR891pqWW \\ uMfbvVi+BXnkIGahoWkMzAQKrLKqOSuNW3 29 \\ GXkd5+5riKEqkYRUehKqv9ygF0vW7g= \\ Welcome in RSA \\ POSThttps://localhost:8443/BlowFishencrypt \\ Welcome in Blowfish Encryption \\ i7fG4zzMXBWy $1 s 6 n 5 \mathrm{G}+\mathrm{u} 5 \mathrm{mK} 70 \mathrm{VTNHM}$ \\ R5Ma9DR3BaAfN/nSERIain $10==$ \\ POST nttps://localhost:8443/BlowFishdecrypt \\ i7fG4zzMXBWy $1 s 6 n 5 \mathrm{G}+\mathrm{u} 5 \mathrm{mK} 70 \mathrm{VTNHM}$ \\ R5Ma9DR3BaAfN/nSERIqjn1Q== \\ Welcome in Blowfish Encryption \\ POST https://localhost:8443/AESencrypt

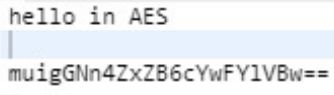


Figure 7

\section{An API based security system}

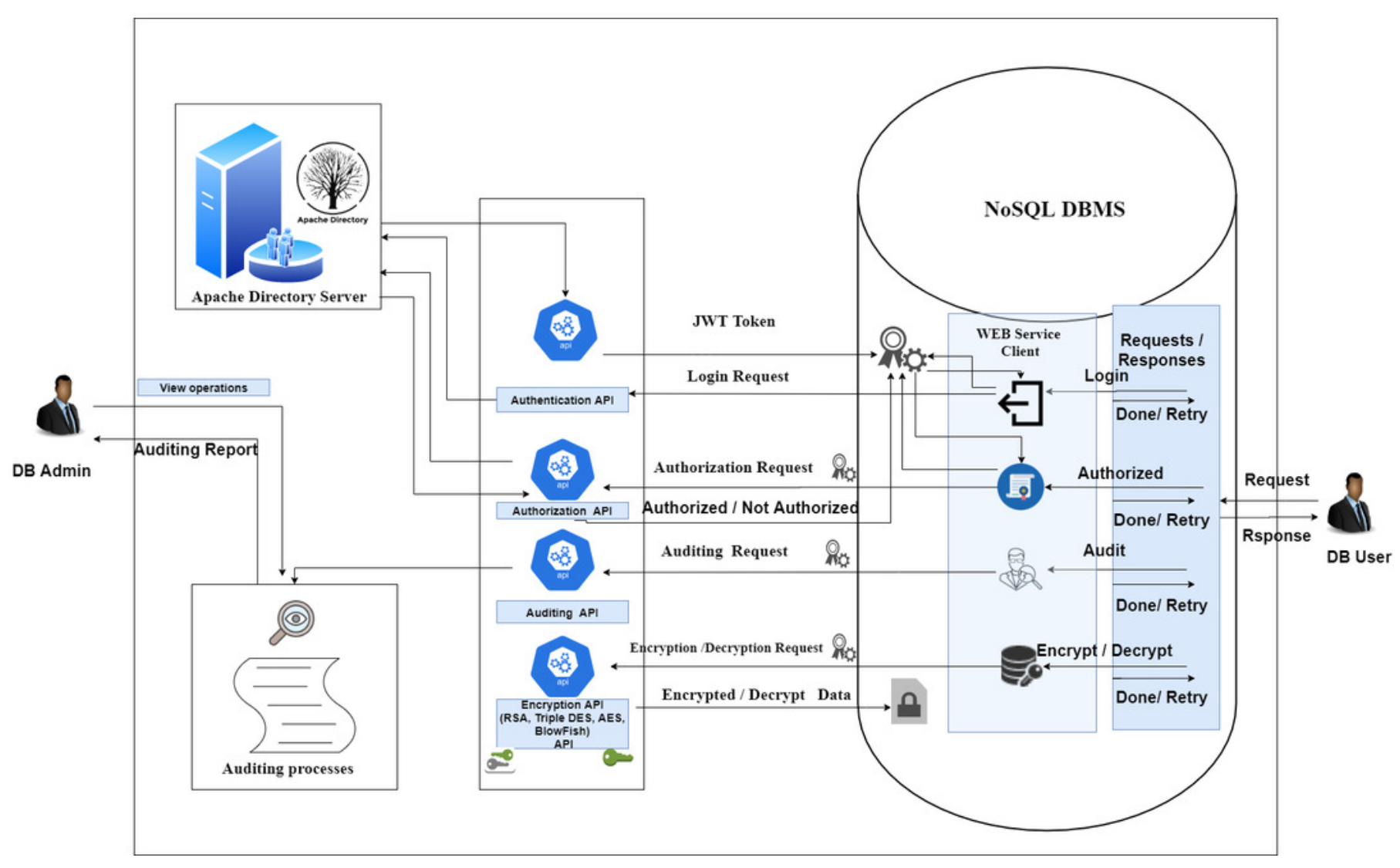

